\title{
Static and Dynamical, Fractional Uncertainty Principles
}

\author{
Kumar, S. ${ }^{* 1}$, Ponce-Vanegas, F. ${ }^{\dagger 1}$, and Vega, L. ${ }^{\ddagger 1,2}$ \\ ${ }^{1}$ BCAM - Basque Center for Applied Mathematics \\ ${ }^{2}$ Universidad del País Vasco UPV/EHU
}

\begin{abstract}
We study the process of dispersion of low-regularity solutions to the Schrödinger equation using fractional weights (observables). We give another proof of the uncertainty principle for fractional weights and use it to get a lower bound for the concentration of mass. We consider also the evolution when the initial datum is the Dirac comb in $\mathbb{R}$. In this case we find fluctuations that concentrate at rational times and that resemble a realization of a Lévy process. Furthermore, the evolution exhibits multifractality.
\end{abstract}

\section{Introduction}

This work grew out of the interest in understanding the process of dispersion of solutions to the Schrödinger equation with initial data with low regularity. By Schrödinger equation we mean the following initial value problem:

$$
\left\{\begin{array}{l}
\partial_{t} u=\frac{i}{2} \hbar \Delta u \\
u(x, 0)=f(x)
\end{array}\right.
$$

\footnotetext{
*skumar@bcamath.org

†fponce@bcamath.org

${ }^{\ddagger}$ lvega@bcamath.org
} 
where $\hbar:=1 /(2 \pi)$.

We measure regularity using the space

$$
\Sigma_{\delta}\left(\mathbb{R}^{n}\right):=\left\{f \in L^{2}\left(\mathbb{R}^{n}\right)\left|\|f\|_{\Sigma_{\delta}}^{2}:=\left\||x|^{\delta} f\right\|_{2}^{2}+\left\|D^{\delta} f\right\|_{2}^{2}<\infty\right\},\right.
$$

where $D^{\delta} f:=|\xi|^{\delta} \hat{f}(\xi)$ and

$$
\hat{f}(\xi):=\int_{\mathbb{R}^{n}} e^{-2 \pi i x \xi} f(x) d x .
$$

We will consider $0<\delta \leqslant 1$, and refer to solutions with $u(x, 0) \in \Sigma_{\delta}\left(\mathbb{R}^{n}\right)$, for $0<\delta<1$, as low-regularity solutions.

Similarly, we measure the dispersion of a solution $u$ with the functional

$$
h_{\delta}[f](t):=\int|x|^{2 \delta}|u(x, t)|^{2} d x
$$

for simplicity, we may write $h_{\delta}(t)$. Nahas and Ponce studied this functional during their work on persistence properties of decay and regularity in the non-linear setting [32. As a consequence of Lemma 2 in [32] we have

$$
h_{\delta}[f](t) \leqslant C_{\delta}\|f\|_{\Sigma_{\delta}\left(\mathbb{R}^{n}\right)}^{2}\left(1+t^{2}\right)^{\delta},
$$

where $f$ is the initial datum, so the functional (2) makes sense for every time. Another proof of this persistence property is given in [1], where the motivation is to give sufficient conditions for uniqueness of linear and nonlinear Schrödinger equations following the ideas in [17.

From another point of view, $h_{\delta}[f](t)$ is the evolution of the average value of a quantum observable and the corresponding quantity for a classical particle in free-motion is $h_{\delta}^{\mathrm{c}}\left[x_{0}, p_{0}\right](t):=\left|x_{0}+p_{0} t\right|^{2 \delta}$, where $x_{0}$ and $p_{0}$ are the initial position and momentum, respectively. It is interesting to compare the quantum and classical behavior; for example, after computing $h_{1}^{\prime \prime}$ or by using the identity $e^{i \pi t|\xi|^{2}}(i \hbar \partial) e^{-i \pi t|\xi|^{2}}=i \hbar \partial+t \xi$ we can see that

$$
h_{1}[f](t)=\left\langle\left(x_{0}+p_{0} t\right)^{2}\right\rangle:=\int f(x) \overline{(x-i t \hbar \partial)^{2} f} d x,
$$

where $x_{0}=x$ and $p_{0}=-i \hbar \partial$ are the initial (in the Heisenberg picture) position and momentum operators, respectively. Does this simple and smooth behavior hold equally when $0<\delta<1$ ? 
The classical Heisenberg's Uncertainty Principle asserts that

$$
\left[\int|x|^{2}|f(x)|^{2} d x \int|\xi|^{2}|\hat{f}(\xi)|^{2} d x\right]^{\frac{1}{2}} \geqslant \frac{n}{4 \pi}\|f\|_{L^{2}\left(\mathbb{R}^{n}\right)}^{2} .
$$

Using translations in physical space and in phase space (i.e. Galilean transformations) it is always possible to assume that $\int x|f(x)|^{2} d x=\int \xi|\hat{f}(\xi)|^{2} d \xi=0$, and (4) is then a measure of the concentration of $|f|$ and $|\hat{f}|$ around the origin. Finally, using translations in time and dilations we can also assume that $\left\langle x_{0} p_{0}+p_{0} x_{0}\right\rangle=0$ and that $a^{2}:=\left\langle x_{0}^{2}\right\rangle=\left\langle p_{0}^{2}\right\rangle$, so in that case $h_{1}[f](t)=a^{2}\left(1+t^{2}\right)$. Hence, using (4) we conclude that if $\|f\|_{L^{2}\left(\mathbb{R}^{n}\right)}=1$ then

$$
h_{1}[f](t) \geqslant \frac{n}{4 \pi}\left(1+t^{2}\right),
$$

and the identity holds if and only if $f=c f_{G}(x):=c 2^{n / 4} e^{-\pi|x|^{2}}$, where $|c|=1$. In fact, in that case the corresponding solution is explicitly given by $u_{G}=$ $2^{n / 4}(1+i t)^{-n / 2} e^{-\pi|x|^{2} /(1+i t)}$, so

$$
h_{\delta}\left[f_{G}\right](t)=h_{\delta}\left[f_{G}\right](0)\left(1+t^{2}\right)^{\delta} .
$$

The above argument suggests that a lower bound of $h_{\delta}[f](t)$ might be proved by means of a generalization of the uncertainty principle (4) with weights $|x|^{2 \delta}$ and $|\xi|^{2 \delta}$, for $0<\delta<1$. As it is well known, the uncertainty principle has been already extended in several directions, see e.g. [12, $\underline{3}$, 18, 4, 29, 37, and the "fractional uncertainty principle" we are interested in was proved by Hirschman in [26]. One of the results in this paper is another proof of this fact.

Theorem 1 (Static, Fractional Uncertainty Principle). There exists a constant $a_{\delta}>0$, for $0<\delta<1$, such that

$$
\inf _{\|f\|_{2}=1}\left\||x|^{\delta} f\right\|_{L^{2}\left(\mathbb{R}^{n}\right)}\left\|D^{\delta} f\right\|_{L^{2}\left(\mathbb{R}^{n}\right)}=a_{\delta}^{2} .
$$

Equality is attained and a minimizer $Q_{\delta}$ can be chosen strictly positive and satisfying $\left\||x|^{\delta} Q_{\delta}\right\|_{2}=\left\|D^{\delta} Q_{\delta}\right\|_{2}$. Any other minimizer $f$ is of the form $f(x)=c \lambda^{n / 2} Q_{\delta}(\lambda x)$ for some $\lambda>0$ and $|c|=1$. Furthermore, $Q_{\delta}(x) \simeq$ $|x|^{-n-4 \delta}$ for $|x| \gg 1$.

The decay result is direct consequence of the work of Kaleta and Kulczycki [31. Observe that the minimizer of the fractional uncertainty principle does not decay exponentially. 
As a consequence of the above theorem we can easily obtain a lower bound for $h_{\delta}[f](t)$ as stated in our next theorem.

Theorem 2 (Dynamical, Fractional Uncertainty Principle). If $f \in \Sigma_{\delta}\left(\mathbb{R}^{n}\right)$, for $0<\delta<1$, and $\|f\|_{2}=1$, then

$$
h_{\delta}[f](t) \geqslant\left(\frac{a_{\delta}^{2}}{\left\||x|^{\delta} f\right\|_{2}\left\|D^{\delta} f\right\|_{2}}\right)^{2} \max \left(\left\||x|^{\delta} f\right\|_{2}^{2},\left\|D^{\delta} f\right\|_{2}^{2}|t|^{2 \delta}\right),
$$

where $a_{\delta}$ is the constant in (6). Furthermore, for any $T \neq 0$

$$
h_{\delta}[f](0) h_{\delta}[f](T) \geqslant a_{\delta}^{4}|T|^{2 \delta},
$$

with equality if and only if

$$
f(x)=c e^{-\pi i|x|^{2} / T} \lambda^{n / 2} Q_{\delta}(\lambda x)
$$

for some $\lambda>0$ and $|c|=1$.

One could wonder up to what extent the behavior exhibited by the gaussian in (5) is generic for $h_{\delta}[f](t)$. One of the main purposes of this paper is to start to explore the answer to this question.

In Section 3 we study the regularity of $h_{\delta}[f](t)$. Theorem 9 is one of our main tools, and in this theorem we compute the Fourier transform of $h_{\delta}[f](t)$ away from zero. Since the weight is $|x|^{2 \delta}$, one may expect that $h_{\delta}$ has Lipschitz regularity $2 \delta$ and that it is best possible in general; in Theorem 11 we confirm this when $n \geqslant 2$. However, when $n=1$ and $\delta>1 / 2$ the Lipschitz regularity drops to $\frac{1}{4}+\frac{3}{2} \delta$.

In Theorem 13 we supplement our results about regularity with the analysis of the decay of $\hat{h}_{\delta}$. In dimension $n \geqslant 4$ we prove that $\left|\hat{h}_{\delta}(\tau)\right| \leqslant \tau^{-1-2 \delta}$, which is consistent with the Lipschitz regularity. However, when $n \leqslant 3$ the Fourier transform decays more slowly.

From the analysis of the Fourier transform of $h_{\delta}$ one can guess that the so called Talbot effect can generate plenty of fluctuations from the generic behavior $\left(1+t^{2}\right)^{\delta}$, so we turn our attention to it.

In the torus, the fundamental solution $u_{D}$ of the Schrödinger equation (when the initial datum is $\delta_{0}$ ) has a very peculiar behavior which influence the evolution of any other function. The Talbot effect might be described as the emergence, at rational times, of equally spaced Dirac deltas in the fundamental solution, each Dirac delta being multiplied by a complex weight 
depending on the Gauss sums. In $\mathbb{R}^{n}$ we can think of $u_{D}$ as the solution when the initial datum is the Dirac comb.

The Talbot effect was discovered by Henry Talbot when experimenting with ray lights crossing a grating with equidistant slits [38]. The effect was theoretically investigated in several papers by Berry and collaborators, e.g. [23, 7, 6], who discovered various interesting properties.

In a deep connection, the Talbot effect appears during the evolution of a polygonal vortex filament in a fluid through the binormal flow $X_{t}=\kappa \boldsymbol{b}$. Here $X$ is an arc-length parametrization of the filament, $\kappa$ is the curvature and $\boldsymbol{b}$ is the binormal vector. In the case of a polygonal filament, the curvature is zero except for the corners where $\kappa$ has Dirac deltas, so $\kappa$ may be seen as a weighted Dirac comb, the weights depending on the angle of the corner. In 25] Hasimoto discovered a transformation that carries a solution of the binormal flow (curvature and torsion) to a solution of the 1D cubic non-linear Schrödinger equation (NLS), so we can use a solution of the NLS with the Dirac comb as initial datum to study the evolution of a polygonal vortex filament. We also notice that a version of the Talbot effect was observed for the NLS numerically [9], and then supported theoretically [14]; see also Ch. 5.3 of [16] or [15, 11] for further information.

In [13] the Talbot effect is exploited to show that a polygonal vortex filament evolves into a polygon at rational times as long as uniqueness holds for the binormal flow. They found compelling numerical evidence that $\int_{0}^{t} u_{D}(0, s) d s$ approximates the trajectory of a corner in a vortex filament, which has found more theoretical support recently [2]. Furthermore, the dynamics displays the same conspicuous fractal nature described by Berry and collaborators with respect to the Talbot effect; see also [34].

Inspired by ideas in theory of turbulence [21], one may wonder whether $\int_{0}^{t} u_{D}(0, s) d s$ can be seen as the outcome of a stochastic process. As far as we know, such a description is still missing; however, as we will show in Section 4.1, when the curves $t \mapsto u_{D}(x, t)$ are "averaged" over $x \in \mathbb{R}$, such a description as a stochastic process might be possible.

Thus, as a second step, we focus our attention to one space dimension and to the particular case when $f$ is the Dirac comb

$$
F_{D}(x):=\sum_{m \in \mathbb{Z}} \delta(x-m)
$$

Even though $F_{D}$ is not a proper function but a distribution, so at first $h_{\delta}\left[F_{D}\right]$ does not make sense, we are able to extend, after renormalization, the func- 

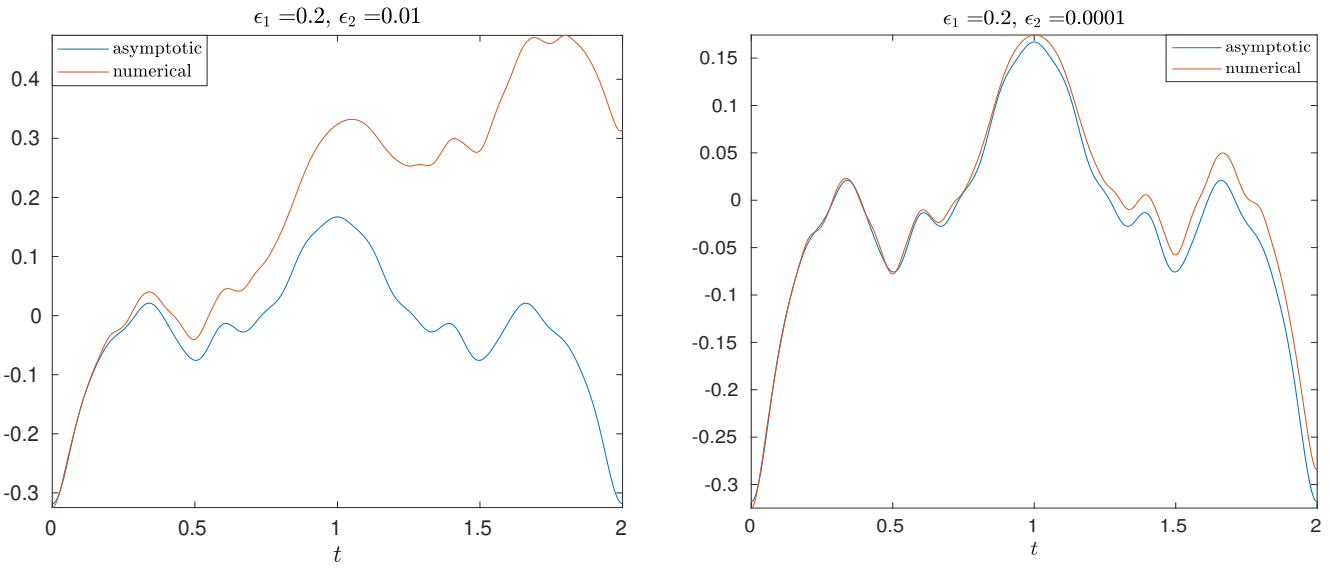

Figure 1: The red line is the plot of $h_{\delta}\left[f_{\varepsilon_{1}, \varepsilon_{2}}\right]$, for $\delta=0.25$, using its definition in (2), and the blue line is $h_{\mathrm{p}, \delta}\left[F_{\varepsilon_{1}}\right]$, to be defined in (43). In this plot we have removed from $h_{\delta}\left[f_{\varepsilon_{1}, \varepsilon_{2}}\right]$ a constant term $C_{\varepsilon_{2}}$ and then multiplied by $\varepsilon_{2}^{-1}$; this will be clear when we reach (52). The choice of $\varepsilon_{1}=0.2$ is due to the high computational cost of taking a smaller value of $\varepsilon_{1}$ and then to diminish $\varepsilon_{2}$.

tional $h_{\delta}$ to periodic functions and then to the Dirac comb. To approach the Dirac comb in $\mathbb{R}$ we use functions of the form

$$
f_{\varepsilon_{1}, \varepsilon_{2}}(x):=N_{\varepsilon_{2}}^{-1} \psi\left(\varepsilon_{2} x\right) F_{\varepsilon_{1}} /\left\|F_{\varepsilon_{1}}\right\|_{2},
$$

where $\psi$ is a smooth function with $\psi(0)=1, N_{\varepsilon_{2}}$ is chosen so that $\left\|f_{\varepsilon_{1}, \varepsilon_{2}}\right\|_{2}=$ 1 , and

$$
F_{\varepsilon_{1}}(x):=\sum_{m \in \mathbb{Z}} \varepsilon_{1}^{-1} e^{-\pi\left((x-m) / \varepsilon_{1}\right)^{2}}=\sum_{m \in \mathbb{Z}} e^{-\pi\left(\varepsilon_{1} m\right)^{2}} e^{2 \pi i x m} .
$$

We will prove that in the limit $\varepsilon_{2} \rightarrow 0$ ( $\varepsilon_{1}$ fixed) the function $h_{\delta}\left[f_{\varepsilon_{1}, \varepsilon_{2}}\right]$ splits into a smooth background and a oscillating, periodic function that we call $h_{\mathrm{p}, \delta}\left[F_{\varepsilon_{1}}\right]$. In Figure 1 we can see how $h_{\delta}\left[f_{\varepsilon_{1}, \varepsilon_{2}}\right]$ approaches, after renormalization, $h_{\mathrm{p}, \delta}\left[F_{\varepsilon_{1}}\right]$.

The final step is to pass to the limit $\varepsilon_{1} \rightarrow 0$. In this way we obtain a periodic, pure point distribution $h_{\mathrm{p}, \delta}\left[F_{D}\right]$ with support at rational times, a fact which is very reminiscent of the Talbot effect. More concretely, we prove the following result; see Fig. 2, 


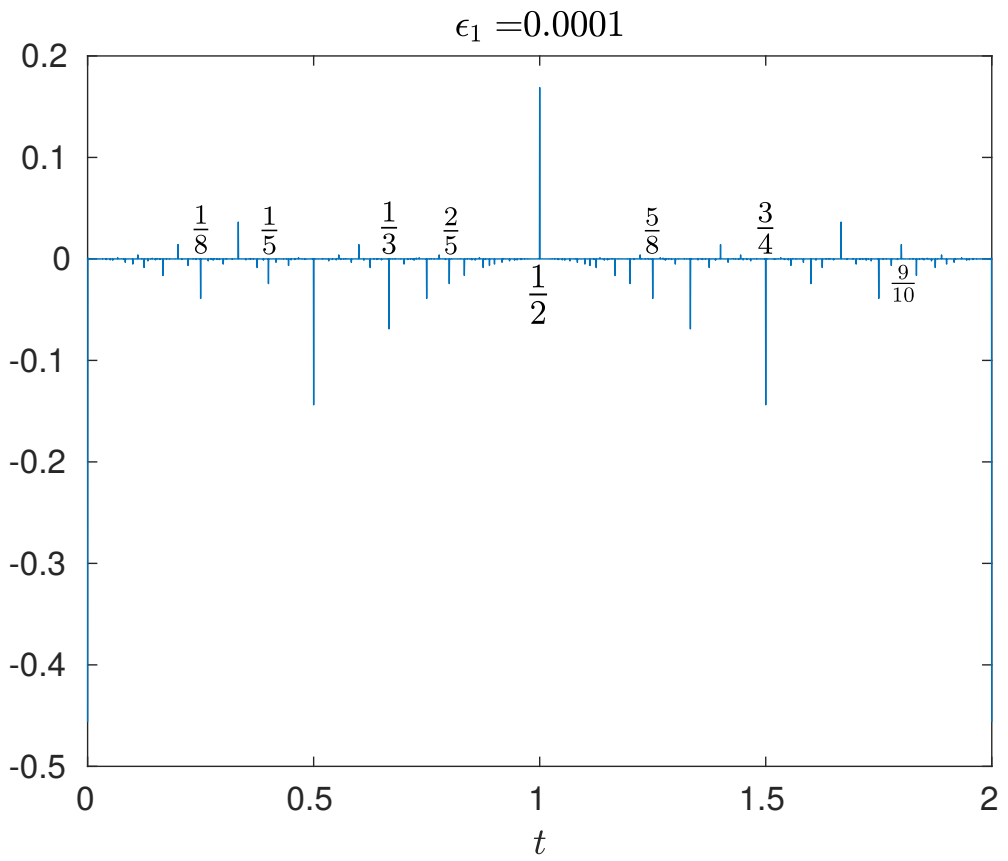

Figure 2: Plot of $h_{\mathrm{p}, \delta}\left[F_{\varepsilon_{1}}\right]$, to be defined in (43), when $\delta=0.25$. In Figure 1 the plot of $h_{\mathrm{p}, \delta}\left[F_{\varepsilon_{1}}\right]$ lacks the rich structure suggested by (8) because $\varepsilon_{1}$ is still small there; however, as $\varepsilon_{1}$ approaches zero the emergence of Dirac deltas is clearly visible.

\section{Theorem 3.}

$$
\begin{aligned}
h_{p, \delta}\left[F_{D}\right](2 t)= & -\frac{2 b_{1, \delta}}{\|\psi\|_{2}^{2}} \zeta(2(1+\delta))\left[\sum_{\substack{(p, q)=1 \\
q>0 \text { odd }}} \frac{1}{q^{2(1+\delta)}} \delta \frac{p}{q}(t)-\right. \\
& \left.-\sum_{\substack{(p, q)=1 \\
q \equiv 2(\bmod 4)}} \frac{2\left(2^{1+2 \delta}-1\right)}{q^{2(1+\delta)}} \delta \frac{p}{q}(t)+\sum_{\substack{(p, q)=1 \\
q \equiv 0(\bmod 4)}} \frac{2^{2(1+\delta)}}{q^{2(1+\delta)}} \delta \frac{p}{q}(t)\right],
\end{aligned}
$$

where $\zeta(s)$ is the Riemann zeta function, and

$$
b_{1, \delta}=\frac{1}{(2 \pi)^{2 \delta}} \frac{\Gamma(2 \delta)}{|\Gamma(-\delta)| \Gamma(\delta)} .
$$

Our final result is about the properties of $h_{\mathrm{p}, \delta}\left[F_{D}\right]$. Let us consider its 


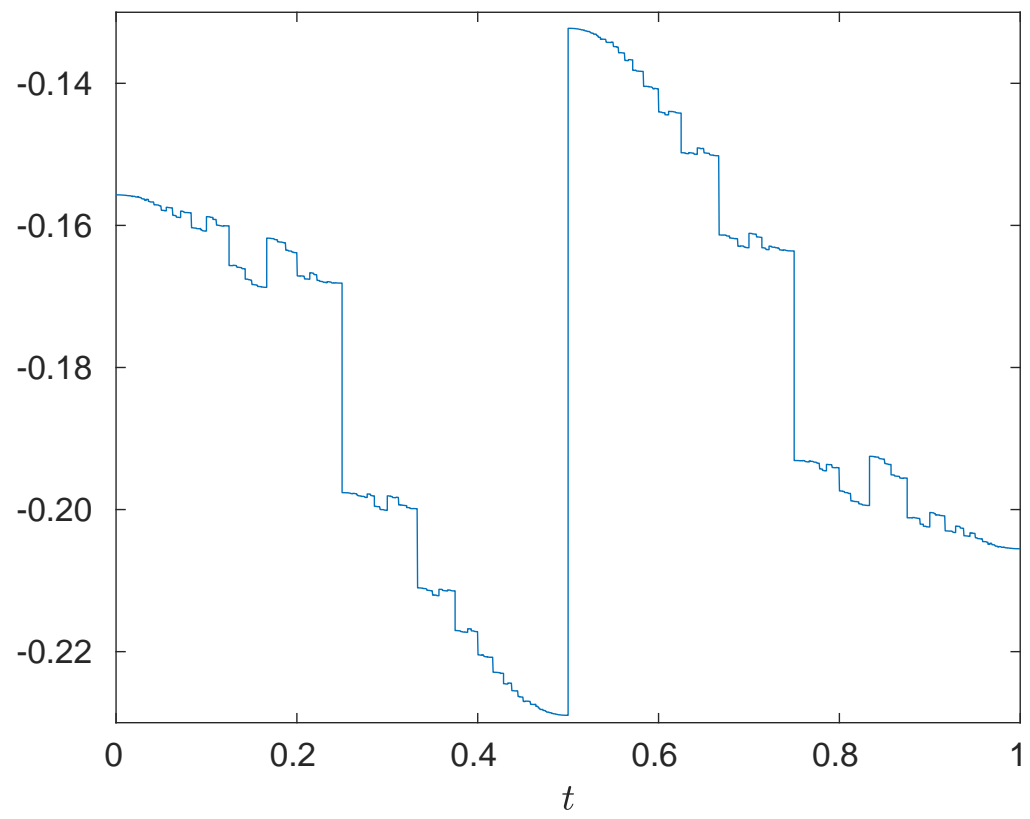

Figure 3: Plot of $H_{\delta}$ in (9). Even though $H_{\delta}$ has some symmetry, e.g. $H_{\delta}(1-t)=c_{\delta}-H_{\delta}(t-)$, the appearance of "unpredictable" large jumps resembles an $\alpha$-Lévy process with small exponent $\alpha$.

primitive, that is,

$$
H_{\delta}(t):=\int_{[0, t]} h_{\mathrm{p}, \delta}(2 s) d s .
$$

As we anticipated when discussing the Talbot effect, $H_{\delta}$ resembles the outcome of a stochastic process, or more precisely, a pure jump $\alpha$-Lévy process with $\alpha:=1 /(1+\delta)$ (see Fig. 3), which strongly suggests the presence of intermittency. We discuss this in more detail in Section 4.1.

Again, inspired by the theory of turbulence (Ch. 8 of [21]), we compute the Hölder exponent of $H_{\delta}$ at each irrational time and show that it depends on its "irrationality" $\mu(t)$; the precise definition of $\mu(t)$ is given in Definition 23 . We also look at the so called spectrum of singularities $d_{H_{\delta}}(\gamma):=\operatorname{dim} F_{\gamma}$, where

$$
F_{\gamma}:=\left\{t \in[0,1) \mid H_{\delta} \text { has Hölder exponent } \gamma \text { at } t\right\} .
$$

By convention $d_{H_{\delta}}(\gamma)=-\infty$ if $F_{\gamma}=\varnothing$. Our main result in this direction is the following one. 
Theorem 4. Let $H_{\delta}$ be the function in (9) and set $\alpha:=1 /(1+\delta)$. Then,

$$
d_{H_{\delta}}(\gamma)= \begin{cases}\alpha \gamma, & \text { if } \gamma \in[0,1 / \alpha] \\ -\infty, & \text { if } \gamma>1 / \alpha\end{cases}
$$

Jaffard proved in Theorem 1 of [28] that the spectrum of singularities of an $\alpha$-Lévy process is almost surely equal to (11). This identity tightens our suggested relationship between $H_{\delta}$ and Lévy processes.

\section{Structure of the paper:}

- In Section 2 we discuss the static, fractional uncertainty principle (Theorem 1I) and prove some properties of the space $\Sigma_{\delta}\left(\mathbb{R}^{n}\right)$.

- In Section 3 we discuss the dynamical, fractional uncertainty principle (Theorem 2) and we compute the Fourier transform of $h_{\delta}[f]$ (Theorem 9). In Section 3.1 we exploit Theorem 9 to obtain regularity properties of $h_{\delta}[f]$.

- In Section 4 we define $h_{\delta}[f]$ for periodic initial data. In Section 4.1 we study the "dispersion" properties of the Dirac comb, and prove Theorems 3 and 4 .

Finally, some questions that arise naturally for future work are:

1. What are the optimal constants in Theorems 1 and 2]? Can $h_{\delta}\left[Q_{\delta}\right]$ be explicitly computed?

2. What are the results about the Dirac Comb in the non-linear setting?

3. Study different regimes for $\varepsilon_{1}$ and $\varepsilon_{2}$ in (7);

4. For other observables (weights) $W(x)$, can we estimate $\left\langle e^{-i t \hbar \Delta / 2} W e^{i t \hbar \Delta / 2}\right\rangle$ in terms of classical trajectories $W(x+t p)$ ?

\section{Notation}

- Relations: If $x \lesssim y$ then $x \leqslant C y$ for some constant $C>0$, and similarly for $x \gtrsim y$ and $x \simeq y$. If $x \ll 1$ then $x \leqslant c$, where $c>0$ is a sufficiently small constant, and similarly for $x \gg 1$.

- Miscellaneous: $a+:=a+\varepsilon$, for $0<\varepsilon \ll 1 .\langle x\rangle:=\left(1+|x|^{2}\right)^{\frac{1}{2}}$. sgn is the sign function. The standard measure on the sphere is denoted by $d S$. 
- If $A \subset \mathbb{R}^{n}$, then $|A|$ is its Lebesgue measure and $\mathbb{1}_{A}$ is the indicator function.

- The fractional derivative is $\left(D^{\delta} f\right)^{\wedge}(\xi):=|\xi|^{\delta} \hat{f}(\xi)$.

- Let $I \subset \mathbb{R}$ be an interval with center $c(I)$. The projection to frequencies $|\xi| \in I$ is the operator $\left(P_{I} f\right)^{\wedge}(\xi):=\zeta_{I} \hat{f}(\xi)$, where $\zeta_{I}(\xi):=\zeta((\xi-$ $c(I)) /|I|)$ and $\zeta$ is a fixed cutoff of $[-1,1]$.

- If $X$ is a function space, then $X_{\text {loc }}:=\left\{f \in \mathcal{S}^{\prime} \mid \zeta f \in X\right.$ for every $\zeta \in$ $\left.C_{0}^{\infty}\right\}$.

- Spaces: for $\Sigma_{\delta}\left(\mathbb{R}^{n}\right)$ see (1), and for $\Lambda^{\alpha}\left(\mathbb{R}^{n}\right)$ see (27). $H^{s}\left(\mathbb{R}^{n}\right)$ is the space of $f \in L^{2}\left(\mathbb{R}^{n}\right)$ with $D^{s} f \in L^{2}\left(\mathbb{R}^{n}\right)$.

- $h_{f}(t)$ is the Hölder exponent of a function $f$ at $t \in \mathbb{R}$; see Def. 222). $d_{f}$ is the spectrum of singularities; see (10).

- $\mu(t)$ is the irrationality measure of $t \in \mathbb{R}$; see Def. 23.

\section{Funding}

This research is supported by the Basque Government through the BERC 2018-2021 program, by the Spanish State Research Agency through BCAM Severo Ochoa excellence accreditation SEV-2017-0718, and by the ERCEA Advanced Grant 2014 669689-HADE. The second author is also supported by the project PGC2018-094528-B-I00.

\section{Acknowledgments}

We thank Daniel Eceizabarrena for many insightful conversations over the course of this work. We also thank the referees for their suggestions, they helped us to improve the exposition of our work.

\section{Static, Fractional Uncertainty Principle}

In this section we study the static, fractional uncertainty principle. We prove some general properties of $\Sigma_{\delta}\left(\mathbb{R}^{n}\right)$, which will play an important role in our investigation of $h_{\delta}$. 
The (static) uncertainty principle asserts that there exists $a_{\delta}>0$ such that

$$
\left\||x|^{\delta} f\right\|_{2}\left\|D^{\delta} f\right\|_{2} \geqslant a_{\delta}^{2}\|f\|_{2}^{2}, \quad \text { for } 0<\delta \leqslant 1 .
$$

Actually, this is equivalent to the continuous embedding $\Sigma_{\delta}\left(\mathbb{R}^{n}\right) \hookrightarrow L^{2}\left(\mathbb{R}^{n}\right)$. In fact, let us define

$$
a_{\delta}^{2}:=\inf _{\|f\|_{2}=1}\left\||x|^{\delta} f\right\|_{2}\left\|D^{\delta} f\right\|_{2}
$$

We can exploit the symmetry $f_{\lambda}(x):=\lambda^{\frac{n}{2}} f(\lambda x)$ to force the condition $\left\||x|^{\delta} f_{\lambda}\right\|_{2}=\left\|D^{\delta} f_{\lambda}\right\|_{2}$ while preserving $\|f\|_{2}=1$, so that

$$
2 a_{\delta}^{2}=\inf _{\substack{\|f\|_{2}=1 \\\left\|\left.x\right|^{\delta} f\right\|_{2}=\left\|D^{\delta} f\right\|_{2}}} 2\left\||x|^{\delta} f\right\|_{2}\left\|D^{\delta} f\right\|_{2} \geqslant \inf _{\|f\|_{2}=1}\|f\|_{\Sigma_{\delta}}^{2}
$$

On the other hand, $2\left\||x|^{\delta} f\right\|_{2}\left\|D^{\delta} f\right\|_{2} \leqslant\|f\|_{\Sigma_{\delta}}^{2}$ implies the reverse inequality $2 a_{\delta}^{2} \leqslant \inf _{\|f\|_{2}=1}\|f\|_{\Sigma_{\delta}}^{2}$, so

$$
2 a_{\delta}^{2}=\inf _{\|f\|_{2}=1}\|f\|_{\Sigma_{\delta}}^{2} .
$$

Lemma 5. The class $\Sigma_{\delta}\left(\mathbb{R}^{n}\right)$ is a Hilbert space compactly embedded in $L^{2}\left(\mathbb{R}^{n}\right)$; in particular,

$$
\|f\|_{2} \leqslant C\left(\left\||x|^{\delta} f\right\|_{2}^{2}+\left\|D^{\delta} f\right\|_{2}^{2}\right)^{\frac{1}{2}} .
$$

Furthermore, there exists a function $Q_{\delta}$ with $\left\|Q_{\delta}\right\|_{2}=1$ such that

$$
\inf _{\|f\|_{2}=1}\|f\|_{\Sigma_{\delta}}=\left\|Q_{\delta}\right\|_{\Sigma_{\delta}}
$$

Proof. We choose a sequence of functions $\left\{f_{n}\right\}_{n}$ with $\left\|f_{n}\right\|_{2}=1$ that minimizes $\|g\|_{\Sigma_{\delta}}$, that is, $\left\|f_{n}\right\|_{\Sigma_{\delta}} \rightarrow \inf _{\|g\|_{2}=1}\|g\|_{\Sigma_{\delta}}$.

By the Fréchet-Kolmogorov theorem, the sequence $\left\{f_{n}\right\}$ will be relatively compact in $L^{2}\left(\mathbb{R}^{n}\right)$ if the following two conditions holds uniformly in $n$ :

$$
\begin{aligned}
& \text { (1) } \int_{|x|>R}\left|f_{n}\right|^{2} d x<\varepsilon, \quad \text { for every } \varepsilon>0 \text { and } R \gg 1 \\
& \text { (2) }\left\|f_{n}(\cdot-h)-f_{n}\right\|_{2}<\varepsilon, \quad \text { for every } \varepsilon>0 \text { and }|h| \ll 1 .
\end{aligned}
$$

The condition (1) follows from

$$
\int_{|x|>R}\left|f_{n}\right|^{2} d x \leqslant R^{-2 \delta} \int|x|^{2 \delta}\left|f_{n}\right|^{2} d x \lesssim R^{-2 \delta} .
$$


The condition (2) follows from

$$
\begin{aligned}
\left\|f_{n}(\cdot-h)-f_{n}\right\|_{2}^{2} & =\int\left|\hat{f}_{n}\right|^{2}\left|e^{-2 \pi i \xi \cdot h}-1\right|^{2} d \xi \\
& \leqslant \int_{|\xi| \leqslant|h|^{-\frac{1}{2}}}+\int_{|\xi|>|h|^{-\frac{1}{2}}}\left|\hat{f}_{n}\right|^{2}\left|e^{-2 \pi i \xi \cdot h}-1\right|^{2} d \xi \\
& \lesssim|h|+|h|^{\delta} .
\end{aligned}
$$

Hence, we can choose a sub-sequence $\left\{f_{n_{k}}\right\}_{k}$ that converges in $L^{2}\left(\mathbb{R}^{n}\right)$ to some function $Q_{\delta} \in L^{2}\left(\mathbb{R}^{n}\right)$ with $\left\|Q_{\delta}\right\|_{2}=1$.

If $\inf _{\|g\|_{2}=1}\|g\|_{\Sigma_{\delta}}=0$ then $\left\||x|^{\delta} f_{n_{k}}\right\|_{2} \rightarrow 0$ and, passing to a sub-sequence if necessary, we see that $f_{n_{k}} \rightarrow 0$ a.e., which contradicts $\left\|Q_{\delta}\right\|_{2}=1$. Thus, $\inf _{\|g\|_{2}=1}\|g\|_{\Sigma_{\delta}}>0$ and $\Sigma_{\delta}\left(\mathbb{R}^{n}\right)$ is continuously embedded in $L^{2}\left(\mathbb{R}^{n}\right)$, which is (12). Incidentally, the proof shows that the ball $\left\{\|g\|_{\Sigma_{\delta}} \leqslant 1\right\}$ is relatively compact in $L^{2}\left(\mathbb{R}^{n}\right)$, so the embedding is compact.

We now prove that $Q_{\delta} \in \Sigma_{\delta}\left(\mathbb{R}^{n}\right)$. Since $\Sigma_{\delta}\left(\mathbb{R}^{n}\right)$ is a Hilbert space, we can pass to a sub-sequence, say $\left\{f_{n_{k}}\right\}_{k}$, that converges weakly to some $f^{*} \in$ $\Sigma_{\delta}\left(\mathbb{R}^{n}\right)$. By (12) every $h \in L^{2}\left(\mathbb{R}^{n}\right)$ defines a continuous linear map $g \mapsto \int g h$ in $\Sigma_{\delta}\left(\mathbb{R}^{n}\right)$, then $\int Q_{\delta} h=\int f^{*} h$ and $Q_{\delta}=f^{*} \in \Sigma_{\delta}\left(\mathbb{R}^{n}\right)$.

The minimizer is the ground state of a differential equation.

Lemma 6. If $\left\|Q_{\delta}\right\|_{\Sigma_{\delta}}=\inf _{\|u\|_{2}=1}\|u\|_{\Sigma_{\delta}}$ and $\left\|Q_{\delta}\right\|_{2}=1$, then

$$
D^{2 \delta} Q_{\delta}+|x|^{2 \delta} Q_{\delta}=2 a_{\delta}^{2} Q_{\delta}
$$

Proof. We take $v \in \Sigma_{\delta}\left(\mathbb{R}^{n}\right)$, with $\|v\|_{2}=1$, orthogonal to $Q_{\delta}$ in $L^{2}\left(\mathbb{R}^{n}\right)$. Let us define $w(\theta):=\cos \theta Q_{\delta}+\sin \theta v$ so that $f(\theta):=\|w(\theta)\|_{\Sigma_{\delta}}^{2}$ has a minimum at $\theta=0$. Since the derivative is

$$
f^{\prime}(\theta)=\sin (2 \theta)\left(\|v\|_{\Sigma_{\delta}}^{2}-2 a_{\delta}^{2}\right)+2 \cos (2 \theta)\left(Q_{\delta}, v\right)_{\Sigma_{\delta}}
$$

then $\left(Q_{\delta}, v\right)_{\Sigma_{\delta}}=0$; considering $\tilde{v}=v /\|v\|_{2}$, we can remove the condition $\|v\|_{2}=1$.

For any $v \in \Sigma_{\delta}\left(\mathbb{R}^{n}\right)$ the function $P v=v-\left(Q_{\delta}, v\right)_{2} Q_{\delta}$ is orthogonal to $Q_{\delta}$ in $L^{2}\left(\mathbb{R}^{n}\right)$, so we have $\left(Q_{\delta}, P v\right)_{\Sigma_{\delta}}=0$ or

$$
\left(Q_{\delta}, v\right)_{\Sigma_{\delta}}=2 a_{\delta}^{2}\left(Q_{\delta}, v\right)_{2}
$$

which is (13). 
By the Perron-Frobenius theorem (see Ch. XIII.12 of [33) the lowest eigenvalue of the operator $D^{2 \delta}+|x|^{2 \delta}$ has multiplicity one and the ground state can be chosen strictly positive. To apply this method we need to know that the heat kernel $e^{-t D^{2 \delta}}$ is positive; see [10] or Lemma A.1 in [20]. Uniqueness implies that $\hat{Q}_{\delta}=Q_{\delta}$ and that $Q_{\delta}$ is radial.

In Corollary 3 of [31, Kaleta and Kulczycki proved that the ground state satisfies $Q_{\delta}(x) \simeq 1 /|x|^{n+4 \delta}(0<\delta<1)$ for $|x| \gg 1$.

We summarize the discussion so far in the following theorem, which was stated in the introduction.

Theorem 1. There exists a constant $a_{\delta}>0$, for $0<\delta<1$, such that

$$
\inf _{\|f\|_{2}=1}\left\||x|^{\delta} f\right\|_{L^{2}\left(\mathbb{R}^{n}\right)}\left\|D^{\delta} f\right\|_{L^{2}\left(\mathbb{R}^{n}\right)}=a_{\delta}^{2} .
$$

Equality is attained and a minimizer $Q_{\delta}$ can be chosen strictly positive and satisfying $\left\||x|^{\delta} Q_{\delta}\right\|_{2}=\left\|D^{\delta} Q_{\delta}\right\|_{2}$. Any other minimizer $f$ is of the form $f(x)=c \lambda^{n / 2} Q_{\delta}(\lambda x)$ for some $\lambda>0$ and $|c|=1$. Furthermore, $Q_{\delta}(x) \simeq$ $|x|^{-n-4 \delta}$ for $|x| \gg 1$.

We now prove a couple of properties of $\Sigma_{\delta}\left(\mathbb{R}^{n}\right)$.

Lemma 7. The space $C_{0}^{\infty}\left(\mathbb{R}^{n}\right)$ is dense in $\Sigma_{\delta}\left(\mathbb{R}^{n}\right)$.

Proof. We choose a symmetric function $\zeta \in C_{0}^{\infty}\left(\mathbb{R}^{n}\right)$ such that $\zeta \geqslant 0$; we might replace $\zeta$ by $\zeta * \zeta$ to assume also that $\hat{\zeta} \geqslant 0$. By dilation and multiplication by a constant, we assume that $\zeta(0)=1$ and $\int \zeta=1$, and we define $\zeta_{\lambda}(x):=\zeta(x / \lambda)$.

First we prove that functions with compact support are dense in $\Sigma_{\delta}\left(\mathbb{R}^{n}\right)$. We fix $\varepsilon>0$ and choose $R \gg_{\varepsilon} 1$ such that $\left\||x|^{\delta}\left(1-\zeta_{R}\right) f\right\|_{2}<\varepsilon$, so we only have to prove that $\left\||\xi|^{\delta}\left(\hat{f}-\left(\zeta_{R} f\right)^{\wedge}\right)\right\|_{2} \lesssim \varepsilon$ for $R \gg 1$.

We choose $\lambda \gg_{\varepsilon} 1$ such that $\left\||\xi|^{\delta} \mathbb{1}_{|\xi|>\lambda} \hat{f}\right\|_{L^{2}}<\varepsilon$. Since $\left(\zeta_{R} f\right)^{\wedge} \rightarrow \hat{f}$ in $L^{2}$, then for $R \gg_{\varepsilon, \lambda} 1$ we have that $\left\||\xi|^{\delta} \mathbb{1}_{|\xi|<2 \lambda}\left[\hat{f}-\left(\zeta_{R} u\right)^{\wedge}\right]\right\|_{2}<\varepsilon$. By Jensen's inequality $\left|\left(\zeta_{R} f\right)^{\wedge}\right|^{2} \leqslant \hat{\zeta}_{R} *|\hat{f}|^{2}$, so

$$
\begin{aligned}
\int_{|\xi|>2 \lambda}|\xi|^{2 \delta}\left|\left(\zeta_{R} f\right)^{\wedge}\right|^{2} d \xi & \leqslant \int\left(|\xi|^{2 \delta} \mathbb{1}_{|\xi|>2 \lambda}\right) * \hat{\zeta}_{R}|\hat{f}|^{2} d \xi \\
& \lesssim \frac{1}{R \lambda} \int_{|\xi|<\lambda}|\hat{f}|^{2} d \xi+\int_{|\xi|>\lambda}|\xi|^{2 \delta}|\hat{f}|^{2} d \xi \\
& \lesssim \frac{1}{R \lambda} \int|\hat{f}|^{2} d \xi+\varepsilon^{2}
\end{aligned}
$$


where we exploited the rapid decay of $\hat{\zeta}_{R}$. Hence, $\left\||\xi|^{\delta} \mathbb{1}_{|\xi|>2 \lambda}\left(\zeta_{R} f\right)^{\wedge}\right\|_{2} \leqslant C \varepsilon$ for $R \gg_{\varepsilon, \lambda} 1$, and

$$
\begin{aligned}
\left\||\xi|^{\delta}\left(\hat{f}-\left(\zeta_{R} f\right)^{\wedge}\right)\right\|_{2} \leqslant \||\xi|^{\delta} & \mathbb{1}_{|\xi|<2 \lambda}\left[\hat{f}-\left(\zeta_{R} f\right)^{\wedge}\right] \|_{2}+ \\
& +\left\||\xi|^{\delta} \mathbb{1}_{|\xi|>2 \lambda} \hat{f}\right\|_{2}+\left\||\xi|^{\delta} \mathbb{1}_{|\xi|>2 \lambda}\left(\zeta_{R} f\right)^{\wedge}\right\|_{2} \leqslant C \varepsilon
\end{aligned}
$$

which shows that functions with compact support are dense in $\Sigma_{\delta}\left(\mathbb{R}^{n}\right)$.

A similar, though simpler argument shows that a function $f \in \Sigma_{\delta}\left(\mathbb{R}^{n}\right)$ with compact support can be approximated by functions $\zeta_{\rho} * f \in C_{0}^{\infty}\left(\mathbb{R}^{n}\right)$.

The next lemma contains some embedding properties.

Lemma 8. If $f \in \Sigma_{\delta}\left(\mathbb{R}^{n}\right)$, then $f, \hat{f} \in H^{\delta}\left(\mathbb{R}^{n}\right) \cap L^{p}\left(\mathbb{R}^{n}\right)$, where $p$ satisfies:

$$
\begin{aligned}
& \frac{1}{2}-\frac{\delta}{n} \leqslant \frac{1}{p}<\frac{1}{2}+\frac{\delta}{n} \quad \text { if } n \geqslant 2 \text {, or } n=1 \text { and } \delta<\frac{1}{2} \text {, } \\
& 0<\frac{1}{p}<1 \quad \text { if } n=1 \text { and } \delta=\frac{1}{2} \text {, } \\
& 0 \leqslant \frac{1}{p} \leqslant 1 \quad \text { if } n=1 \text { and } \delta>\frac{1}{2} \text {. }
\end{aligned}
$$

Proof. The inequalities at the left follow from the Sobolev Embedding Theorem, and those at the right follow from Hölder inequality.

We cannot improve the strict inequalities in (15), and we can use the examples $f(x):=\zeta(|x|)|x|^{-\frac{n}{2}-\delta}(\log |x|)^{-\frac{1}{2}-\varepsilon}$, for $0<\varepsilon<\delta / n$, where $\zeta \in$ $C^{\infty}(\mathbb{R})$ vanishes around zero. When $n=1$ and $\delta=1 / 2$, it is known that $f$ may not be bounded.

\section{Dynamical, Fractional Uncertainty Principle}

In this section we turn our attention to $h_{\delta}[f]$ in (2). We begin with a lower bound for $h_{\delta}[f]$, and then we compute the Fourier transform of $h_{\delta}[f]$ away from the origin. In Section 3.1 we determine the Hölder regularity of $h_{\delta}$ and the rate of decay of $\hat{h}_{\delta}$.

Theorem 2. If $f \in \Sigma_{\delta}\left(\mathbb{R}^{n}\right)$, for $0<\delta<1$, and $\|f\|_{2}=1$, then

$$
h_{\delta}[f](t) \geqslant\left(\frac{a_{\delta}^{2}}{\left\||x|^{\delta} f\right\|_{2}\left\|D^{\delta} f\right\|_{2}}\right)^{2} \max \left(\left\||x|^{\delta} f\right\|_{2}^{2},\left\|D^{\delta} f\right\|_{2}^{2}|t|^{2 \delta}\right),
$$


where $a_{\delta}$ is the constant in (14). Furthermore, for any $T \neq 0$

$$
h_{\delta}[f](0) h_{\delta}[f](T) \geqslant a_{\delta}^{4}|T|^{2 \delta},
$$

with equality if and only if

$$
f(x)=c e^{-\pi i|x|^{2} / T} \lambda^{n / 2} Q_{\delta}(\lambda x)
$$

for some $\lambda>0$ and $|c|=1$.

Proof. The solution $u$ can be represented as

$$
u(x, t)=\frac{1}{(i t)^{\frac{n}{2}}} e^{\pi i|x|^{2} / t} \int f(y) e^{\pi i|y|^{2} / t-2 \pi i x \cdot y / t} d y, \quad \text { where } \operatorname{Re} \sqrt{i t}>0 .
$$

If we define $g_{t}(y):=f(y) e^{\pi i|y|^{2} / t}$, then the solution can be written as

$$
u(x, t)=\frac{1}{(i t)^{\frac{n}{2}}} e^{\pi i|x|^{2} / t} \hat{g}_{t}(x / t) .
$$

By the uncertainty principle (14) we have

$$
a_{\delta}^{2} \leqslant\left\||x|^{\delta} g_{t}\right\|_{2}\left\|D^{\delta} g_{t}\right\|_{2}=|t|^{-\delta} h_{\delta}(0)^{\frac{1}{2}} h_{\delta}(t)^{\frac{1}{2}},
$$

with equality if and only if $g_{t}(x)=c \lambda^{n / 2} Q_{\delta}(\lambda x)$ for some $\lambda>0$ and $|c|=1$, so (17) and (18) hold. This inequality implies the lower bound

$$
h_{\delta}(t) \geqslant \frac{a_{\delta}^{4}}{\left\|\left.x\right|^{\delta} f\right\|_{2}^{2}}|t|^{2 \delta} .
$$

On the other hand, again by (14), we have

$$
a_{\delta}^{4} \leqslant h_{\delta}(t) \int|\xi|^{2 \delta}|\hat{u}(\xi, t)|^{2} d \xi=h_{\delta}(t) \int|\xi|^{2 \delta}|\hat{f}(\xi)|^{2} d \xi,
$$

which implies the lower bound

$$
h_{\delta}(t) \geqslant \frac{a_{\delta}^{4}}{\left\|D^{\delta} f\right\|_{2}^{2}} .
$$

From (19) and (20) we conclude that

$$
h_{\delta}(t) \geqslant \max \left(\frac{a_{\delta}^{4}}{\left\|D^{\delta} f\right\|_{2}^{2}}, \frac{a_{\delta}^{4}}{\left\||x|^{\delta} f\right\|_{2}^{2}}|t|^{2 \delta}\right),
$$

which is (16) after reordering. 
The computation of the Fourier transform of $h_{\delta}[f]$ is motivated by the oscillations that appear in numerical simulations when $f$ approaches the Dirac comb.

Theorem 9. If $f \in \Sigma_{\delta}\left(\mathbb{R}^{n}\right)$, then the Fourier transform of $h_{\delta}[f]$ in $\mathbb{R} \backslash\{0\}$ can be represented as

$$
\hat{h}_{\delta}(\tau)=-2 b_{n, \delta} \int_{\mathbb{R}^{2 n}} \hat{f}(\xi) \overline{\hat{f}}(\eta) \delta_{0}\left(\tau-\frac{|\eta|^{2}-|\xi|^{2}}{2}\right) \frac{d \xi d \eta}{|\xi-\eta|^{n+2 \delta}},
$$

where

$$
b_{n, \delta}=\frac{1}{2 \pi^{n / 2+2 \delta}} \frac{\Gamma\left(\frac{n+2 \delta}{2}\right)}{|\Gamma(-\delta)|} .
$$

If $\varphi \in \mathcal{S}(\mathbb{R})$ is supported outside the interval $(-a, a)$, then

$$
\left|\left\langle\hat{h}_{\delta}[f], \varphi\right\rangle\right| \leqslant C_{a}\|f\|_{\Sigma_{\delta}}^{2}\|\varphi\|_{\infty}
$$

Furthermore,

$$
\left\|\hat{h}_{\delta}[f]\right\|_{L^{1}(\mathbb{R} \backslash[-a, a])} \leqslant C_{a}\|f\|_{\Sigma_{\delta}}^{2} .
$$

Proof. The computation is based on the identity (see [19])

$$
h_{\delta}(t)=b_{n, \delta} \int_{\mathbb{R}^{n} \times \mathbb{R}^{n}} \frac{\left|e^{-\pi i t|\xi|^{2}} \hat{f}(\xi)-e^{-\pi i t|\eta|^{2}} \hat{f}(\eta)\right|^{2}}{|\xi-\eta|^{n+2 \delta}} d \xi d \eta, \quad \text { for } 0<\delta<1 .
$$

Let $\varphi \in \mathcal{S}(\mathbb{R})$ be a test function that vanishes in the interval $(-a, a)$. We apply Fubini to write the Fourier transform of $h_{\delta}$ as

$$
\begin{aligned}
\left\langle h_{\delta}, \hat{\varphi}\right\rangle= & b_{n, \delta} \int_{\mathbb{R}^{2 n}} \int\left[|\hat{f}(\xi)|^{2}\right. \\
+ & |\hat{f}(\eta)|^{2}-e^{-\pi i t\left(|\xi|^{2}-|\eta|^{2}\right)} \hat{f}(\xi) \overline{\hat{f}}(\eta)- \\
& \left.-e^{-\pi i t\left(|\eta|^{2}-|\xi|^{2}\right)} \overline{\hat{f}}(\xi) \hat{f}(\eta)\right] \hat{\varphi}(t) d t \frac{d \xi d \eta}{|\xi-\eta|^{n+2 \delta}} \\
=-b_{n, \delta} \int_{\mathbb{R}^{2 n}}\left[\hat{f}(\xi) \overline{\hat{f}}(\eta) \varphi\left(\frac{|\eta|^{2}-|\xi|^{2}}{2}\right)+\right. & \\
& \left.+\overline{\hat{f}}(\xi) \hat{f}(\eta) \varphi\left(\frac{|\xi|^{2}-|\eta|^{2}}{2}\right)\right] \frac{d \xi d \eta}{|\xi-\eta|^{n+2 \delta}}, \quad
\end{aligned}
$$

We have to show that this integral represents a bounded functional in $\mathcal{S}(\mathbb{R})$. 
Assume that $a \leqslant 1$. We bound the integral (24) as

$$
\begin{aligned}
\left|\left\langle h_{\delta}, \hat{\varphi}\right\rangle\right| & \leqslant C \int\left|\hat{f}(\xi) \bar{f}(\eta) \varphi\left(\frac{|\eta|^{2}-|\xi|^{2}}{2}\right)\right| \frac{d \xi d \eta}{|\xi-\eta|^{n+2 \delta}} \\
& \lesssim \int|\hat{f}(\xi)|^{2}\left[\int \varphi\left(\frac{|\eta|^{2}-|\xi|^{2}}{2}\right) \frac{d \eta}{|\xi-\eta|^{n+2 \delta}}\right] d \xi+ \\
& \quad+\int|\hat{f}(\eta)|^{2}\left[\int \varphi\left(\frac{|\eta|^{2}-|\xi|^{2}}{2}\right) \frac{d \xi}{|\xi-\eta|^{n+2 \delta}}\right] d \eta \\
& :=C \int|\hat{f}(\xi)|^{2} J(\xi) d \xi+C \int|\hat{f}(\eta)|^{2} J^{\prime}(\eta) d \eta
\end{aligned}
$$

where $J$ and $J^{\prime}$ are the integrals in square brackets.

We only bound the first integral in (25) , the other being analogous; recall that $\varphi\left(\left(|\eta|^{2}-|\xi|^{2}\right) / 2\right)=0$ if $\left.|| \eta\right|^{2}-|\xi|^{2} \mid<2 a$. When $|\xi|>\sqrt{a}$ we control $J$ as

$$
J(\xi) \leqslant\|\varphi\|_{\infty} \int_{\mathbb{R}^{n} \backslash B(\xi, a /(2|\xi|))} \frac{d \eta}{|\xi-\eta|^{n+2 \delta}} \leqslant C a^{-2 \delta}\|\varphi\|_{\infty}|\xi|^{2 \delta},
$$

and when $|\xi|<\sqrt{a}$ we integrate instead over $\mathbb{R}^{n} \backslash B(\xi, \sqrt{a} / 2)$. The final result is

$$
J(\xi) \leqslant C\|\varphi\|_{\infty} \begin{cases}a^{-2 \delta}|\xi|^{2 \delta} & |\xi|>\sqrt{a} \\ a^{-\delta} & |\xi|<\sqrt{a} .\end{cases}
$$

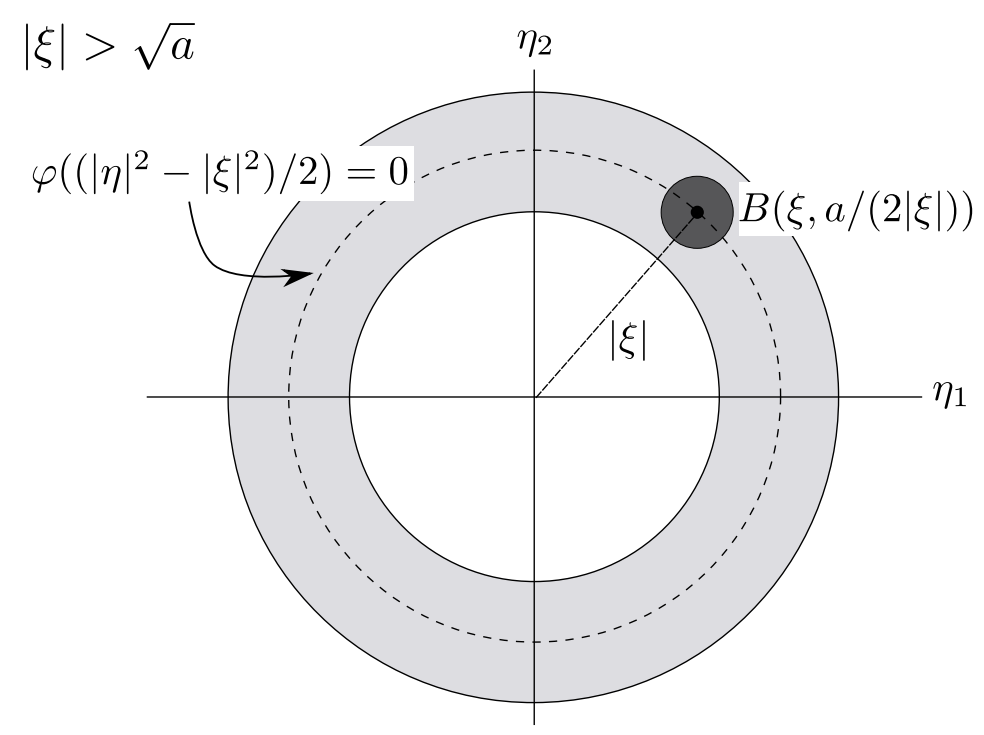


We replace (26) in (25) and use the inclusion $\Sigma_{\delta} \hookrightarrow L^{2}$ to conclude that

$$
\left|\left\langle h_{\delta}, \hat{\varphi}\right\rangle\right| \leqslant C a^{-2 \delta}\|\varphi\|_{\infty}\|f\|_{\Sigma_{\delta}}^{2},
$$

which is (22).

Since $\mathcal{S}(\mathbb{R})$ is dense in the space of continuous functions that vanish at infinity, then from (22) and the Riesz-Markov Theorem we can see $\hat{h}_{\delta}$ as a (signed) regular measure in $\mathbb{R} \backslash[-a, a]$ with total variation $\leqslant C_{a}\|f\|_{\Sigma_{\delta}}^{2}$.

The measure $\hat{h}_{\delta}$ is actually an $L^{1}$-function away from the origin. If $U \subset$ $\mathbb{R} \backslash[-a, a]$ is an open set, then we can approximate monotonically $\mathbb{1}_{U}$ with Schwartz functions $\varphi$ such that $0 \leqslant \varphi \leqslant 1$ and $\operatorname{supp} \varphi \subset U$, so by dominated convergence we can write

$$
\left\langle\hat{h}_{\delta}, \mathbb{1}_{U}\right\rangle=-2 b_{n, \delta} \int_{\mathbb{R}^{2 n}} \hat{f}(\xi) \hat{f}(\eta) \mathbb{1}_{U}\left(\frac{|\eta|^{2}-|\xi|^{2}}{2}\right) \frac{d \xi d \eta}{|\xi-\eta|^{n+2 \delta}} .
$$

Since $\hat{h}_{\delta}$ is a regular measure, we can actually extend this identity from $\mathbb{1}_{U}$ to all bounded, Borel measurable functions. If $A \subset \mathbb{R} \backslash[-a, a]$ is a bounded, Borel set with $|A|=0$, then we can apply this identity to $\psi \mathbb{1}_{A}$, for $|\psi| \leqslant 1$, to conclude that $\hat{h}_{\delta}$ is absolutely continuous away from the origin.

Corollary 10. The function $h_{\delta}$ is continuous.

Proof. We split $h_{\delta}$ into $P_{<1} h_{\delta}$ (an analytic function) and $P_{>1} h_{\delta}$. By (23) $\left(P_{>1} h_{\delta}\right)^{\wedge} \in L^{1}(\mathbb{R})$ and the claim follows.

\subsection{Regularity of $h_{\delta}$}

Corollary 10 says that $h_{\delta}$ is continuous; however, we can improve our estimates and refine the information about regularity.

The space of Lipschitz functions $\Lambda^{\alpha}\left(\mathbb{R}^{n}\right)$, for $\alpha>0$, is

$$
\begin{aligned}
\Lambda^{\alpha}\left(\mathbb{R}^{n}\right):=\left\{f \in L^{\infty}\left(\mathbb{R}^{n}\right) \mid\right. & \\
& \left.\left\|P_{\left[2^{k}, 2^{k+1}\right]} f\right\|_{\infty} \leqslant C 2^{-\alpha k}, \text { for } k \geqslant 0, \text { and }\left\|P_{[0,1]} f\right\|_{\infty} \leqslant C\right\} .
\end{aligned}
$$

If $f \in \Lambda^{\alpha}(\mathbb{R})$, for $0<\alpha<1$, then $|f(x)-f(y)| \leqslant C|x-y|^{\alpha}$; see Ch. V.4 of [35. 
Theorem 11. If $f \in \Sigma_{\delta}\left(\mathbb{R}^{n}\right)$, for $0<\delta<1$, then

$$
\left\|\psi h_{\delta}[f]\right\|_{\Lambda^{\alpha}} \lesssim C_{\psi}\|f\|_{\Sigma_{\delta}}^{2}
$$

where $\psi \in C_{0}^{\infty}(\mathbb{R})$ and

$$
\alpha= \begin{cases}2 \delta & \text { for } n \geqslant 2, \text { or for } n=1 \text { and } \delta<\frac{1}{2}, \\ 1- & \text { for } n=1 \text { and } \delta=\frac{1}{2}, \\ \frac{1}{4}+\frac{3}{2} \delta & \text { for } n=1 \text { and } \delta>\frac{1}{2} .\end{cases}
$$

The result is the best possible - up to the end point in the case $n=1$ and $\delta=\frac{1}{2}$. In particular, $h_{\delta} \in C_{\text {loc }}^{1}(\mathbb{R})$ when $\delta>\frac{1}{2}$.

Proof. Since $P_{\leqslant 1} h_{\delta}$ and its derivatives are bounded in compact sets by the Nahas-Ponce inequality (3), then it suffices to prove that $P_{\geqslant 1} h_{\delta} \in \Lambda^{\alpha}(\mathbb{R})$. Since $h_{\delta}$ is real, then $\hat{h}_{\delta}(\tau)=\overline{\hat{h}}_{\delta}(-\tau)$ and we only need to work with positive frequencies. Hence, by the Hausdorff-Young inequality, it suffices to prove

$$
\left\|\hat{h}_{\delta}\right\|_{L^{1}\left(\tau \simeq 2^{k}\right)} \leqslant C\|f\|_{\Sigma_{\delta}}^{2} \begin{cases}2^{-2 \delta k} & \text { for } n \geqslant 2, \text { or for } n=1 \text { and } \delta \leqslant \frac{1}{2} \\ 2^{-\left(\frac{1}{4}+\frac{3}{2} \delta\right) k} & \text { for } n=1 \text { and } \delta>\frac{1}{2} .\end{cases}
$$

We define $I_{\lambda}:=[\lambda, 2 \lambda]$, for $\lambda \geqslant 1$, and re-scale (24) to get, for $|g| \leqslant 1$,

$$
\left|\left\langle\hat{h}_{\delta}, g \mathbb{1}_{I_{\lambda}}\right\rangle\right| \leqslant C \lambda^{\frac{n}{2}-\delta} \int_{\mathbb{R}^{2 n}}|\hat{f}(\sqrt{\lambda} \xi) \hat{f}(\sqrt{\lambda} \eta)| \mathbb{1}_{I}\left(\frac{|\eta|^{2}-|\xi|^{2}}{2}\right) \frac{d \xi d \eta}{|\xi-\eta|^{n+2 \delta}}
$$

where $I=[1,2]$.

To bound the integral over the region $\{|\xi|>1\}$, we begin with

$$
\begin{aligned}
\left|\left\langle\hat{h}_{\delta}, g \mathbb{1}_{I_{\lambda}}\right\rangle\right|\{|\xi|>1\} & \leqslant C \lambda^{\frac{n}{2}-\delta} \int_{|\xi|>1}|\hat{f}(\sqrt{\lambda} \xi)|^{2}\left[\int \mathbb{1}_{I \cup-I}\left(\frac{|\eta|^{2}-|\xi|^{2}}{2}\right) \frac{d \eta}{|\xi-\eta|^{n+2 \delta}}\right] d \xi \\
& :=C \lambda^{\frac{n}{2}-\delta} \int_{|\xi|>1}|\hat{f}(\sqrt{\lambda} \xi)|^{2} J(\xi) d \xi ;
\end{aligned}
$$

compare with (25). We use (26)), for $a=1$, to find out

$$
\begin{aligned}
\left|\left\langle\hat{h}_{\delta}, g \mathbb{1}_{I_{\lambda}}\right\rangle\right|_{\{|\xi|>1\}} & \leqslant C \lambda^{\frac{n}{2}-\delta} \int|\hat{f}(\sqrt{\lambda} \xi)|^{2}|\xi|^{2 \delta} d \xi \\
& \leqslant C \lambda^{-2 \delta}\|f\|_{\Sigma_{\delta}}^{2} .
\end{aligned}
$$


To bound the integral over the region $\{|\xi|<1\}$, we begin with (29) and notice that the factor $\mathbb{1}_{I}\left(\left(|\eta|^{2}-|\xi|^{2}\right) / 2\right)$ forces $|\eta| \simeq 1$. Hence,

$$
\begin{aligned}
\left|\left\langle\hat{h}_{\delta}, g \mathbb{1}_{I_{\lambda}}\right\rangle\right|\{|\xi|<1\} & \leqslant C \lambda^{\frac{n}{2}-\delta} \int_{|\xi|<1,|\eta| \simeq 1}|\hat{f}(\sqrt{\lambda} \xi) \hat{f}(\sqrt{\lambda} \eta)| d \xi d \eta \\
& \leqslant C \lambda^{-\frac{n}{2}-\delta} \int_{|\xi|<\sqrt{\lambda}}|\hat{f}(\xi)| d \xi \int_{|\eta| \simeq \sqrt{\lambda}}|\hat{f}(\eta)| d \eta \\
& \leqslant C \lambda^{-\frac{n}{4}-\frac{3}{2} \delta}\left(\int_{|\xi|<\sqrt{\lambda}}|\hat{f}(\xi)| d \xi\right)\left\||\eta|^{\delta} \hat{f}\right\|_{2} .
\end{aligned}
$$

We control the term in parentheses as

$$
\begin{aligned}
\int_{|\xi|<\sqrt{\lambda}}|\hat{f}(\xi)| d \xi \lesssim( & \left.\int_{|\xi|<1}|\hat{f}(\xi)|^{2} d \xi\right)^{\frac{1}{2}}+ \\
& +\left(\int_{1<|\xi|<\sqrt{\lambda}}|\xi|^{-2 \delta} d \xi\right)^{\frac{1}{2}}\left(\int_{1<|\xi|<\sqrt{\lambda}}|\xi|^{2 \delta}|\hat{f}(\xi)|^{2} d \xi\right)^{\frac{1}{2}}
\end{aligned}
$$

after replacing in (31) we arrive to

$$
\left|\left\langle\hat{h}_{\delta}, g \mathbb{1}_{I_{\lambda}}\right\rangle\right|_{\{|\xi|<1\}} \lesssim\|f\|_{\Sigma_{\delta}}^{2} \begin{cases}\lambda^{-2 \delta} & \text { for } n \geqslant 2 \text {, or } n=1 \text { and } \delta<\frac{1}{2}, \\ \lambda^{-1} \sqrt{\log \lambda} & \text { for } n=1 \text { and } \delta=\frac{1}{2}, \\ \lambda^{-\frac{1}{4}-\frac{3}{2} \delta} & \text { for } n=1 \text { and } \delta>\frac{1}{2},\end{cases}
$$

which together with (30) implies (28) —notice that $\frac{1}{4}+\frac{3}{2} \delta<2 \delta$.

Sharpness of the regularity

We consider functions $\hat{f}_{\alpha}(\xi):=\langle\xi\rangle^{-\alpha}$, for $\alpha=\frac{n}{2}+\delta+$. The Fourier transform of $h_{\delta}[f]$ is symmetric and, for $\tau>0$, it equals

$$
\hat{h}_{\delta}(\tau)=-2 b_{n, \delta} \tau^{\frac{n}{2}-1-\delta} \int \hat{f}_{\alpha}(\sqrt{\tau} \xi) \hat{f}_{\alpha}(\sqrt{\tau} \eta) \delta\left(1-\frac{|\eta|^{2}-|\xi|^{2}}{2}\right) \frac{d \xi d \eta}{|\xi-\eta|^{n+2 \delta}}
$$

Since $\hat{h}_{\delta} \leqslant 0$, it is enough to prove that $\left|\hat{h}_{\delta}(\tau)\right| \geqslant c \tau^{-1-\beta}$ for $|\tau| \gg 1$, where

$$
\beta= \begin{cases}2 \delta+ & \text { for } n \geqslant 2, \text { or for } n=1 \text { and } \delta<\frac{1}{2} \\ \frac{1}{4}+\frac{3}{2} \delta+ & \text { for } n=1 \text { and } \delta \geqslant \frac{1}{2} .\end{cases}
$$

In fact, if $\left\{\zeta_{I}\right\}$ is a cut-off function of $I:=\left\{2^{k} \leqslant|\tau| \leqslant 2^{k+1}\right\}$, then

$$
c 2^{-\beta k} \leqslant\left\|\zeta_{I} \hat{h}_{\delta}\right\|_{1}=\left|P_{I} h_{\delta}(0)\right| \leqslant\left\|P_{I} h_{\delta}\right\|_{\infty},
$$


and $\hat{h}_{\delta} \notin \Lambda^{\beta+}(\mathbb{R})_{\text {loc }}$.

We use spherical coordinates and bound $\hat{h}_{\delta}$ from below as

$$
\begin{aligned}
\left|\hat{h}_{\delta}(\tau)\right| \geqslant c \tau^{\frac{n}{2}-1-\delta} \int_{\tau^{-\frac{1}{2}}<r_{1}<1} \hat{f}_{\alpha}\left(\sqrt{\tau} r_{1}\right) \hat{f}_{\alpha}\left(\sqrt{\tau} r_{2}\right) \delta\left(1-\frac{r_{2}^{2}-r_{1}^{2}}{2}\right) & \\
& {\left[\int_{S^{n-1} \times S^{n-1}} \frac{d \theta_{1} d \theta_{2}}{\left|r_{1} \theta_{1}-r_{2} \theta_{2}\right|^{n+2 \delta}}\right] r_{1}^{n-1} r_{2}^{n-1} d r_{1} d r_{2} . }
\end{aligned}
$$

We denote by $J\left(r_{1}, r_{2}\right)$ the term inside parentheses; by rotational symmetry

$$
J\left(r_{1}, r_{2}\right)=c \int_{S^{n-1}} \frac{d \theta}{\left|r_{1} e_{n}-r_{2} \theta\right|^{n+2 \delta}} .
$$

The term $\delta\left(1-\left(r_{2}^{2}-r_{1}^{2}\right) / 2\right)$ forces $r_{2} \simeq 1$, so $J\left(r_{1}, r_{2}\right) \gtrsim 1$, and from (33) we deduce

$$
\begin{aligned}
\left|\hat{h}_{\delta}(\tau)\right| & \geqslant c \tau^{\frac{n}{2}-1-\delta} \int_{\tau^{-\frac{1}{2}}}^{1} \frac{1}{\tau^{\alpha} r_{1}^{\alpha}} \int_{0}^{\infty} \delta\left(1-\frac{r_{2}^{2}-r_{1}^{2}}{2}\right) d r_{2} r_{1}^{n-1} d r_{1} \\
& \geqslant c \tau^{\frac{n}{2}-1-\delta-\alpha} \int_{\tau^{-\frac{1}{2}}}^{1} r_{1}^{n-1-\alpha} d r_{1}
\end{aligned}
$$

Since $\alpha=\frac{n}{2}+\delta+$, we conclude, for $|\tau| \gg 1$, that

$$
\left|\hat{h}_{\delta}(\tau)\right| \geqslant c \begin{cases}\tau^{-1-2 \delta-} & \text { for } n \geqslant 2, \text { or for } n=1 \text { and } \delta<\frac{1}{2} \\ \tau^{-1-\frac{1}{4}-\frac{3}{2} \delta-} & \text { for } n=1 \text { and } \delta \geqslant \frac{1}{2},\end{cases}
$$

which implies (32).

As a final remark, if $f$ is one of the examples we used, then $h_{\delta}[f]$, which is an even function, has a singularity at zero of the form $|t|^{\rho}$. By translation in time, we can place the singularity at any other time.

We may compare the regularity of $h_{\delta}$ with its classical counterpart $h_{\delta}^{\mathrm{c}}[x, \xi](t):=$ $|x+t \xi|^{2 \delta}$, which belongs to $\Lambda_{\text {loc }}^{2 \delta}(\mathbb{R})$. If $n \geqslant 2$ then $h_{\delta}^{\mathrm{c}}$ is smooth for a general choice of $x$ and $\xi$, but if $n=1$ then $h_{\delta}^{\mathrm{c}}$ has a singularity for a general choice, which agrees with the loss of regularity in Theorem 11 when $n=1$.

In the following theorem we investigate the rate of decay of $\hat{h}_{\delta}$. However, first we have to prove an auxiliary result. 
Lemma 12. Let $n \geqslant 1$ and let $r_{1}$ and $r_{2}$ be different, positive numbers. If $\alpha>n-1$ and $A, B \in L^{2}\left(S^{n-1}\right)$, then

$$
\int_{S^{n-1} \times S^{n-1}} \frac{A\left(\theta_{1}\right) B\left(\theta_{2}\right) d \theta_{1} d \theta_{2}}{\left|r_{1} \theta_{1}-r_{2} \theta_{2}\right|^{\alpha}} \leqslant C_{r_{1}, r_{2}}\|A\|_{L^{2}\left(S^{n-1}\right)}\|B\|_{L^{2}\left(S^{n-1}\right)}
$$

where

$$
C_{r_{1}, r_{2}} \lesssim \begin{cases}1 / r_{1}^{\alpha} & \text { for } r_{1}>2 r_{2}, \\ 1 / r_{2}^{\alpha} & \text { for } r_{2}>2 r_{1}, \\ \left(r_{1} r_{2}\right)^{-\frac{n-1}{2}}\left|r_{1}-r_{2}\right|^{n-1-\alpha} & \text { for } \frac{1}{2} r_{2}<r_{1}<2 r_{2}\end{cases}
$$

Proof. We assume that $r_{2}<r_{1}$ and that $\|A\|_{2}=\|B\|_{2}=1$, so

$$
\begin{aligned}
\int \frac{A\left(\theta_{1}\right) B\left(\theta_{2}\right) d \theta_{1} d \theta_{2}}{\left|r_{1} \theta_{1}-r_{2} \theta_{2}\right|^{\alpha}} & \leqslant \frac{1}{2} \sup _{\theta_{1}} \int \frac{d \theta_{2}}{\left|r_{1} \theta_{1}-r_{2} \theta_{2}\right|^{\alpha}}+\frac{1}{2} \sup _{\theta_{2}} \int \frac{d \theta_{1}}{\left|r_{1} \theta_{1}-r_{2} \theta_{2}\right|^{\alpha}} \\
& =\frac{1}{r_{2}^{\alpha}} \int_{S^{n-1}} \frac{d \theta}{\left|\rho \theta-e_{n}\right|^{\alpha}}
\end{aligned}
$$

where $\rho:=r_{1} / r_{2}>1$.

When $\rho \geqslant 2$, we notice that $\left|\rho \theta-e_{n}\right| \geqslant \rho / 2$, so

$$
\int \frac{d \theta}{\left|\rho \theta-e_{n}\right|^{\alpha}} \leqslant C \frac{1}{\rho^{\alpha}}
$$

which implies $C_{r_{1}, r_{2}} \lesssim 1 / r_{1}^{\alpha}$, for $r_{1}>2 r_{2}$.

When $\rho<2$, we notice that

$$
\begin{aligned}
\left|\rho\left(\theta-e_{n}\right)+(\rho-1) e_{n}\right|^{2} & =2 \rho^{2}\left(\theta_{n}-1\right)^{2}+(\rho-1)^{2}+2 \rho(\rho-1)\left(\theta_{n}-1\right) \\
& \geqslant 2 \rho^{2}\left(\theta_{n}-1\right)^{2}+(\rho-1)^{2}-a \rho^{2}\left(\theta_{n}-1\right)^{2}-a^{-1}(\rho-1)^{2},
\end{aligned}
$$

so we can take either $a=2$ or $a=1$ to see $\left|\rho \theta-e_{n}\right| \geqslant c \max \left\{\rho\left|\theta-e_{n}\right|, \rho-1\right\}$. Hence,

$$
\begin{aligned}
\int \frac{d \theta}{\left|\rho \theta-e_{n}\right|^{\alpha}} & \lesssim \rho^{-\alpha} \int_{\rho\left|\theta-e_{n}\right|>\rho-1} \frac{d \theta}{\left|\theta-e_{n}\right|^{\alpha}}+(\rho-1)^{-\alpha} \int_{\rho\left|\theta-e_{n}\right|<\rho-1} d \theta \\
& \simeq(\rho-1)^{n-1-\alpha} / \rho^{n-1},
\end{aligned}
$$

which implies $C_{r_{1}, r_{2}} \lesssim\left|r_{1}-r_{2}\right|^{n-1-\alpha}\left(r_{1} r_{2}\right)^{-\frac{n-1}{2}}$, for $r_{2}<r_{1}<2 r_{2}$. 
Theorem 13. If $f \in \Sigma_{\delta}\left(\mathbb{R}^{n}\right)$, for $0<\delta<1$, then for $|\tau| \geqslant 1$ it holds

$$
\left|\hat{h}_{\delta}[f](\tau)\right| \leqslant C\|f\|_{\Sigma_{\delta}}^{2} \begin{cases}\tau^{-1-2 \delta} & \text { for } n \geqslant 3 \text { and } \delta \leqslant \frac{n}{2}-1, \\ \tau^{-\frac{n+2}{4}-\frac{3}{2} \delta} & \text { for } n=2,3 \text { and } \delta>\frac{n}{2}-1, \quad \text { a.e. } \\ \tau^{-\frac{3}{4}-\frac{3}{2} \delta+} & \text { for } n=1 .\end{cases}
$$

The rate of decay is the best possible - up to the end point when $n=1$.

Theorem 13 provides an alternative proof of Theorem 11 when $n \geqslant 3$ and $\delta \leqslant \frac{n}{2}-1$.

Proof. We can assume that $f \in C_{0}^{\infty}\left(\mathbb{R}^{n}\right)$. In fact, for general $f \in \Sigma_{\delta}\left(\mathbb{R}^{n}\right)$ we can take a sequence of functions $\left\{f_{n}\right\}_{n}$ in $C_{0}^{\infty}\left(\mathbb{R}^{n}\right)$ (Lemma 7) converging to $f$ in $\Sigma_{\delta}\left(\mathbb{R}^{n}\right)$. If we reprise the arguments in the proof of (23) we can see that

$$
\|\hat{h}[f]-\hat{h}[g]\|_{L^{1}(\mathbb{R} \backslash[-1,1])} \leqslant C\|f-g\|_{\Sigma_{\delta}}\left(\|f\|_{\Sigma_{\delta}}+\|g\|_{\Sigma_{\delta}}\right) .
$$

Thus, passing to a sub-sequence $h_{\delta}\left[f_{n}\right] \rightarrow h_{\delta}[f]$ a.e. and we are done.

Since $\hat{h}_{\delta}(-\tau)=\overline{\hat{h}}_{\delta}(\tau)$, we assume $\tau>0$. We re-scale (21) to write

$$
\hat{h}_{\delta}(\tau)=-2 b_{n, \delta} \tau^{\frac{n}{2}-\delta-1} \int \hat{f}(\sqrt{\tau} \xi) \overline{\hat{f}}(\sqrt{\tau} \eta) \delta\left(1-\frac{|\eta|^{2}-|\xi|^{2}}{2}\right) \frac{d \xi d \eta}{|\xi-\eta|^{n+2 \delta}}
$$

passing to spherical coordinates we have

$$
\begin{aligned}
\left|\hat{h}_{\delta}(\tau)\right| \leqslant C \tau^{\frac{n}{2}-\delta-1} \int \delta & \left(1-\frac{r_{2}^{2}-r_{1}^{2}}{2}\right) r_{1}^{n-1} r_{2}^{n-1} \\
& {\left[\int_{S^{n-1} \times S^{n-1}} \frac{\left|\hat{f}\left(\sqrt{\tau} r_{1} \theta_{1}\right) \hat{f}\left(\sqrt{\tau} r_{2} \theta_{2}\right)\right| d \theta_{1} d \theta_{2}}{\left|r_{1} \theta_{1}-r_{2} \theta_{2}\right|^{n+2 \delta}}\right] d r_{1} d r_{2} . }
\end{aligned}
$$

The term $\delta\left(1-\left(r_{2}^{2}-r_{1}^{2}\right) / 2\right)$ forces $\left|r_{2} / r_{1}\right|=\sqrt{1+2 / r_{1}^{2}}$.

We apply Lemma 12 to the term in parentheses to deduce

$$
\begin{aligned}
\left|\hat{h}_{\delta}(\tau)\right| \leqslant C \tau^{\frac{n}{2}-\delta-1} \int \delta & \left(1-\frac{r_{2}^{2}-r_{1}^{2}}{2}\right) r_{1}^{n-1} r_{2}^{n-1}\left\|\hat{f}\left(\sqrt{\tau} r_{1} \cdot\right)\right\|_{2}\left\|\hat{f}\left(\sqrt{\tau} r_{2} \cdot\right)\right\|_{2} \\
& {\left[\mathbb{1}_{\left\{r_{1}>\sqrt{2 / 3}\right\}} \frac{1}{\left(r_{1} r_{2}\right)^{\frac{n}{2}-1-\delta}}+\mathbb{1}_{\left\{r_{1}<\sqrt{2 / 3}\right\}} \frac{1}{r_{2}^{n+2 \delta}}\right] d r_{1} d r_{2} } \\
:=I_{\left\{r_{1}>\sqrt{2 / 3}\right\}}+ & I_{\left\{r_{1}<\sqrt{2 / 3}\right\}} .
\end{aligned}
$$


We bound the contribution over the region $\left\{r_{1}>\sqrt{2 / 3}\right\}$ as

$$
\begin{aligned}
I_{\left\{r_{1}>\sqrt{2 / 3}\right\}} \leqslant C \tau^{\frac{n}{2}-\delta-1} \int_{r_{1}>\sqrt{2 / 3}} \delta\left(1-\frac{r_{2}^{2}-r_{1}^{2}}{2}\right) \\
\quad\left[r_{1}^{n+2 \delta}\left\|\hat{f}\left(\sqrt{\tau} r_{1} \cdot\right)\right\|_{2}^{2}+r_{2}^{n+2 \delta}\left\|\hat{f}\left(\sqrt{\tau} r_{2} \cdot\right)\right\|_{2}^{2}\right] d r_{1} d r_{2} \\
\leqslant C \tau^{\frac{n}{2}-\delta-1} \int_{r>c} r^{n-1+2 \delta}\|\hat{f}(\sqrt{\tau} r \cdot)\|_{2}^{2} d r \\
\leqslant C \tau^{-1-2 \delta}\left\||\xi|^{\delta} \hat{f}\right\|_{2}^{2} .
\end{aligned}
$$

It remains to control the integral over $\left\{r_{1}<\sqrt{2 / 3}\right\}$.

When $r_{1}<c$, the term $\delta\left(1-\left(r_{2}^{2}-r_{1}^{2}\right) / 2\right)$ forces $r_{2} \simeq 1$, so

$$
I_{\left\{r_{1}<\sqrt{2 / 3}\right\}} \leqslant C \tau^{\frac{n}{2}-\delta-1} \int_{r_{1}<\sqrt{2 / 3}} r_{1}^{n-1}\left\|\hat{f}\left(\sqrt{\tau} r_{1} \cdot\right)\right\|_{2}\left\|\hat{f}\left(\sqrt{\tau}\left(2+r_{1}^{2}\right)^{\frac{1}{2}} \cdot\right)\right\|_{2} d r_{1}
$$

We leave aside momentarily the case $n=1$. We use Hölder to get (we write $\left.r=r_{1}\right)$

$$
\begin{aligned}
I_{\left\{r_{1}<\sqrt{2 / 3}\right\}} \leqslant C \tau^{\frac{n}{2}-\delta-1}\left(\int_{r<c} r^{2(n-1)-1}\|\hat{f}(\sqrt{\tau} r \cdot)\|_{2}^{2} d r\right)^{\frac{1}{2}} & \\
& \left(\int_{r<c} r\left\|\hat{f}\left(\sqrt{\tau}\left(2+r^{2}\right)^{\frac{1}{2}} \cdot\right)\right\|_{2}^{2} d r\right)^{\frac{1}{2}},
\end{aligned}
$$

after the change of variable $t=\sqrt{2+r^{2}}$ we get

$$
\begin{aligned}
I_{\left\{r_{1}<\sqrt{2 / 3}\right\}} & \leqslant C \tau^{\frac{n}{2}-\delta-1}\left(\int_{|\xi|<c}|\xi|^{n-2}|\hat{f}(\sqrt{\tau} \xi)|^{2} d \xi\right)^{\frac{1}{2}}\left(\int_{|\xi| \simeq 1}|\hat{f}(\sqrt{\tau} \xi)|^{2} d \xi\right)^{\frac{1}{2}} \\
& \leqslant C \tau^{\frac{n}{4}-\frac{3}{2} \delta-1}\left(\int_{|\xi|<c}|\xi|^{n-2}|\hat{f}(\sqrt{\tau} \xi)|^{2} d \xi\right)^{\frac{1}{2}}\left\||\xi|^{\delta} \hat{f}\right\|_{2} .
\end{aligned}
$$

If $n-2 \geqslant 2 \delta$, then we bound the last integral in parentheses as

$$
\int_{|\xi|<c}|\xi|^{n-2}|\hat{f}(\sqrt{\tau} \xi)|^{2} d \xi \leqslant C \int_{|\xi|<c}|\xi|^{2 \delta}|\hat{f}(\sqrt{\tau} \xi)|^{2} d \xi \leqslant C \tau^{-\frac{n}{2}-\delta}\left\||\xi|^{\delta} \hat{f}\right\|_{2}^{2},
$$

so $I_{\left\{r_{1}<\sqrt{2 / 3}\right\}} \leqslant C \tau^{-2 \delta-1}$, which together with (35) and (36) implies the first case in (34). 
If $0 \leqslant n-2<2 \delta$, then

$$
\begin{aligned}
\int_{|\xi|<c}|\xi|^{n-2}|\hat{f}(\sqrt{\tau} \xi)|^{2} d \xi & \leqslant \tau^{-n+1} \int_{|\xi|<\sqrt{\tau} c}|\xi|^{n-2}|\hat{f}(\xi)|^{2} d \xi \\
& \leqslant C \tau^{-n+1}\|f\|_{\Sigma_{\delta}}^{2} .
\end{aligned}
$$

Hence $I_{\left\{r_{1}<\sqrt{2 / 3}\right\}} \leqslant C \tau^{-\frac{n+2}{4}-\frac{3}{2} \delta}$, which together with (35) and (36) implies the second case in (34).

Now we consider the case $n=1$, so we have to bound the integral

$$
I_{\{r<\sqrt{2 / 3}\}}^{\prime}:=C \tau^{-\frac{1}{2}-\delta} \int_{r<c}|\hat{f}(\sqrt{\tau} r)|\left|\hat{f}\left(\sqrt{\tau}\left(2+r^{2}\right)^{\frac{1}{2}}\right)\right| d r .
$$

We intend to use the embedding $\Sigma_{\delta} \hookrightarrow L^{p}$ in Lemma 8. We apply Hölder inequality (twice) and the change of variables $t=\sqrt{2+r^{2}}$ to get

$$
\begin{aligned}
& I_{\{r<\sqrt{2 / 3}\}}^{\prime} \leqslant C \tau^{-\frac{1}{2}-\delta}\left(\int_{r<c}|\hat{f}(\sqrt{\tau} r)|^{p} d r\right)^{\frac{1}{p}}\left(\int_{\sqrt{2}}^{\sqrt{2+c^{2}}}|\hat{f}(\sqrt{\tau} t)|^{p^{\prime}} \frac{d t}{\sqrt{t^{2}-2}}\right)^{\frac{1}{p^{\prime}}} \\
& \leqslant C_{\varepsilon} \tau^{-\frac{1}{2}-\delta-\frac{1}{2 p}}\|\hat{f}\|_{p}\left(\int_{t \simeq 1}|\hat{f}(\sqrt{\tau} t)|^{(2+\varepsilon) p^{\prime}} d t\right)^{\frac{1}{(2+\varepsilon) p^{\prime}}} .
\end{aligned}
$$

If $\delta<\frac{1}{2}$, then we take $\frac{1}{(2+\varepsilon) p^{\prime}}=\frac{1}{2}-\delta$, in which case $\frac{1}{p}=(2+\varepsilon) \delta-\varepsilon / 2<$ $\frac{1}{2}+\delta$, so that

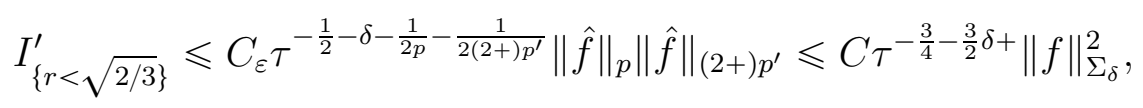

which together with (35) and (36) implies the third case in (34), for $\delta<\frac{1}{2}$.

If $\delta \geqslant \frac{1}{2}$, then we take $p$ very large and notice that

$$
\left(\int_{t \simeq 1}|\hat{f}(\sqrt{\tau} t)|^{(2+\varepsilon) p^{\prime}} d t\right)^{\frac{1}{(2+\varepsilon) p^{\prime}}} \leqslant\left(\int_{t \simeq 1}|\hat{f}(\sqrt{\tau} t)|^{2} d t\right)^{\frac{\theta}{2}}\left(\int|\hat{f}(\sqrt{\tau} t)|^{p} d t\right)^{\frac{1-\theta}{p}}
$$

where $\frac{1}{(2+) p^{\prime}}=\frac{\theta}{2}+\frac{1-\theta}{p}$, so $0<\theta<1$ can be made arbitrarily close to 1 if $p \gg 1$. Hence,

$$
\begin{aligned}
I_{\{r<\sqrt{2 / 3}\}}^{\prime} & \leqslant C_{\varepsilon} \tau^{-\frac{1}{2}-\delta-\frac{2-\theta}{2 p}}\|\hat{f}\|_{p}^{2-\theta}\left(\int_{t \simeq 1}|\hat{f}(\sqrt{\tau} t)|^{2} d t\right)^{\frac{\theta}{2}} \\
& \leqslant C_{\varepsilon} \tau^{-\frac{3}{4}-\frac{3}{2} \delta+}\|\hat{f}\|_{p}^{2-\theta}\left\||\xi|^{\delta} \hat{f}\right\|_{2}^{\theta} \\
& \leqslant C \tau^{-\frac{3}{4}-\frac{3}{2} \delta+}\|f\|_{\Sigma_{\delta}}^{2},
\end{aligned}
$$


which together with (35) and (36) concludes the proof of the last case in (34).

Sharpness of the rate of decay

The example used in Theorem 11 shows that the decay $|\tau|^{-1-2 \delta}$, for $f \in \Sigma_{\delta}$, cannot be improved, so we turn to the case $n \leqslant 3$.

Let $\zeta \in C_{0}^{\infty}(\mathbb{R})$ be a symmetric cut-off of $B_{1}$, and let $d S_{k}$ denote the standard measure on the sphere with radius $2^{k}$ and center at the origin. To construct the example, we define $\zeta_{k}(\xi):=2^{k(n-1)} \zeta\left(2^{k} \xi\right)$ and set

$$
\hat{f}(\xi):=\zeta(\xi)+\sum_{k \geqslant 1} 2^{-k\left(\frac{n-2}{2}+\delta\right)} \frac{1}{k^{2}}\left(\zeta_{k} * d S_{k}\right)(\xi) .
$$

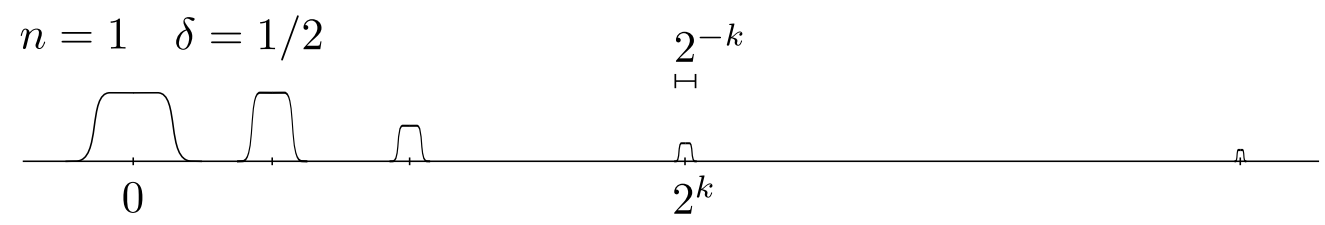

Direct computation shows that $\left\||\xi|^{\delta} \hat{f}\right\|_{2}<\infty$, so we must show that $\left\||x|^{\delta} f\right\|_{2}<$ $\infty$; we only consider the harder case $n \geqslant 2$.

By the triangle inequality

$$
\left\||x|^{\delta} f\right\|_{2} \leqslant\left\||x|^{\delta} \check{\zeta}\right\|_{2}+\sum_{k \geqslant 1} 2^{-k\left(\frac{n-2}{2}+\delta\right)} \frac{1}{k^{2}}\left\||x|^{\delta} \check{\zeta}_{k}\left(d S_{k}\right)^{\vee}\right\|_{2},
$$

After the dilation $x \mapsto 2^{-k} x$, each term in the sum gets into

$$
\left\||x|^{\delta} \check{\zeta}_{k}\left(d S_{k}\right)^{\vee}\right\|_{2}=2^{k\left(\frac{n}{2}-2-\delta\right)}\left\||x|^{\delta} \check{\zeta}\left(2^{-2 k} x\right)(d S)^{\vee}\right\|_{2},
$$

From the inequality $\left|(d S)^{\vee}(\xi)\right| \lesssim\langle\xi\rangle^{-\frac{n-1}{2}}$ [36, Ch. VIII-3] we deduce that $\left\||x|^{\delta} \check{\zeta}_{k}\left(d S_{k}\right)^{\vee}\right\|_{2} \lesssim 2^{k\left(\frac{n-2}{2}+\delta\right)}$, which leads to $\left\||x|^{\delta} f\right\|_{2}<\infty$.

We estimate now $\left|\hat{h}_{\delta}(\tau)\right|$ for $\tau=2^{2 k-1}$ and $k \gg 1$ :

$$
\begin{aligned}
\left|\hat{h}_{\delta}(\tau)\right| & \geqslant c \frac{1}{k^{2}} 2^{-k\left(\frac{n-2}{2}+\delta\right)} \int_{\mathbb{R}^{2 n}} \zeta(\xi)\left(\zeta_{k} * d S_{k}\right)(\eta) \delta\left(\tau-\frac{\eta^{2}-\xi^{2}}{2}\right) \frac{d \xi d \eta}{|\xi-\eta|^{n+2 \delta}} \\
& \geqslant c \frac{1}{k^{2}} 2^{-k\left(\frac{n-2}{2}+\delta\right)} \int_{\mathbb{R}^{2}} \zeta\left(r_{1}\right) \zeta\left(2^{k}\left(r_{2}-\sqrt{2 s}\right)\right) \delta\left(\tau-\frac{r_{2}^{2}-r_{1}^{2}}{2}\right) \frac{r_{1}^{n-1} d r_{1} d r_{2}}{r_{2}^{1+2 \delta}} \\
& \geqslant c \frac{1}{k^{2}} 2^{-k\left(\frac{n-2}{2}+\delta\right)} \tau^{-1-\delta} \int_{\mathbb{R}^{2}} \zeta\left(r_{1}\right) \zeta\left(2^{k}\left(\sqrt{2 s+r_{1}^{2}}-\sqrt{2 s}\right)\right) r_{1}^{n-1} d r_{1} .
\end{aligned}
$$




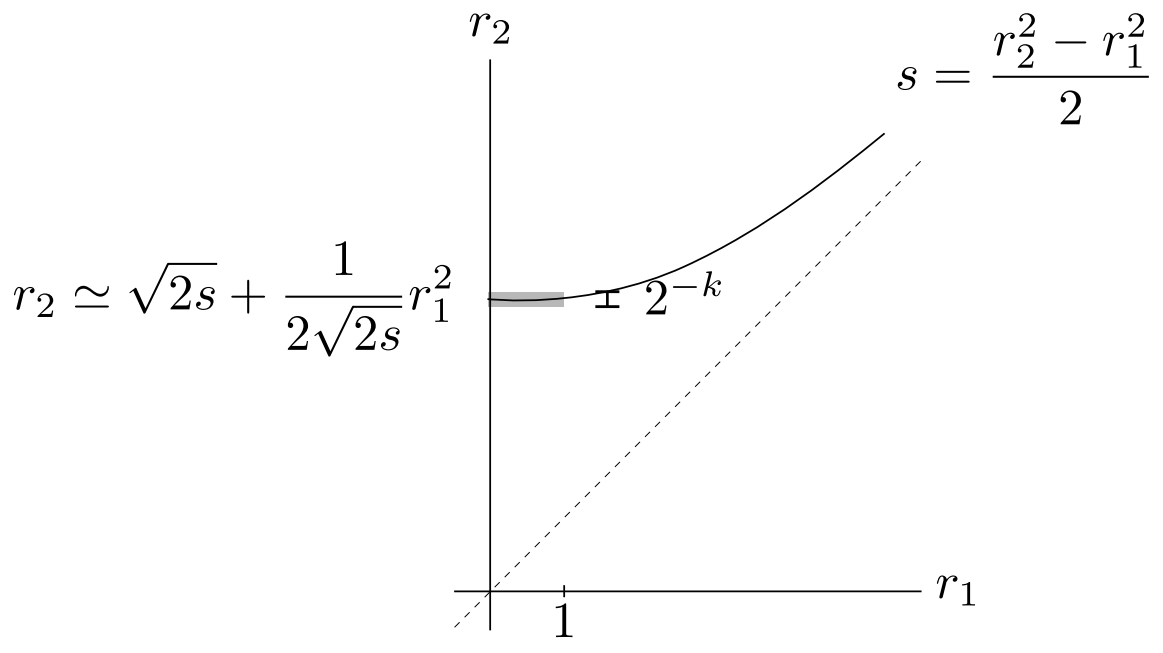

Since $\zeta\left(2^{k}\left(\sqrt{2 s+r_{1}^{2}}-\sqrt{2 s}\right)\right) \gtrsim 1$ for $|\xi|<c$, then

$$
\left|\hat{h}_{\delta}(\tau)\right| \geqslant c \frac{1}{k^{2}} \tau^{-\frac{n+2}{4}-\frac{3}{2} \delta} .
$$

Hence, if $\left|\hat{h}_{\delta}(\tau)\right| \leqslant C \tau^{-\alpha}$, then $\alpha \leqslant \frac{n+2}{4}+\frac{3}{2} \delta$ and the rate of decay in (34) cannot be improved.

\section{Periodic Data}

In the section we extend the definition of $h_{\delta}$ to solutions of the Schrödinger equation with periodic initial data, with the aim to define $h_{\delta}[f]$ when $f$ is the Dirac comb.

We choose a real, symmetric function $\psi \in \mathcal{S}\left(\mathbb{R}^{n}\right)$ with supp $\hat{\psi} \subset B_{1}$ and $\psi(0)=1$. Now we approach a periodic function $F$ in $\mathbb{R}^{n} / \mathbb{Z}^{n}$ as

$$
f_{\varepsilon}(x):=N_{\varepsilon}^{-1} \psi(\varepsilon x) F(x)=N_{\varepsilon}^{-1} \psi(\varepsilon x) \sum_{\nu \in \mathbb{Z}^{n}} \hat{F}(\nu) e(2 \pi i x \cdot \nu),
$$

where $N_{\varepsilon}^{2}=\varepsilon^{-n}\|\psi\|_{2}^{2}\|F\|_{L^{2}(\mathbb{T})}^{2}$ is the normalization constant; henceforth, we will assume that $\|F\|_{L^{2}(\mathbb{T})}=1$. The Fourier transform is

$$
\hat{f}_{\varepsilon}(\xi)=N_{\varepsilon}^{-1} \frac{1}{\varepsilon^{n}} \sum_{\nu \in \mathbb{Z}^{n}} \hat{F}(\nu) \hat{\psi}((\xi-\nu) / \varepsilon) .
$$

We want to study how $h_{\delta}\left[f_{\varepsilon}\right]$ evolves as $\varepsilon \rightarrow 0$. 
The Fourier transform of $h_{\delta}\left[f_{\varepsilon}\right]$ away from the origin is

$$
\begin{aligned}
\left\langle\hat{h}_{\delta}, \varphi\right\rangle= & -2 b_{n, \delta} \int_{\mathbb{R}^{2 n}} \hat{f}_{\varepsilon}(\xi) \overline{\hat{f}}_{\varepsilon}(\eta) \varphi\left(\frac{|\eta|^{2}-|\xi|^{2}}{2}\right) \frac{d \xi d \eta}{|\xi-\eta|^{n+2 \delta}} \\
= & -2 b_{n, \delta} \frac{N_{\varepsilon}^{-2}}{\varepsilon^{2 n}} \sum_{\nu_{1}, \nu_{2}} \hat{F}\left(\nu_{1}\right) \overline{\hat{F}}\left(\nu_{2}\right) \\
& \int \hat{\psi}\left(\left(\xi-\nu_{1}\right) / \varepsilon\right) \hat{\psi}\left(\left(\eta-\nu_{2}\right) / \varepsilon\right) \varphi\left(\frac{|\xi|^{2}-|\eta|^{2}}{2}\right) \frac{d \xi d \eta}{|\xi-\eta|^{n+2 \delta}}
\end{aligned}
$$

where $\varphi \in \mathcal{S}(\mathbb{R})$ is supported away from the origin. In this expression we can distinguish two types of terms: diagonal $\left(\nu_{1}=\nu_{2}\right)$ and off-diagonal $\left(\nu_{1} \neq \nu_{2}\right)$. Diagonal terms are more related to the behavior of $h_{\delta}$ in the large, and offdiagonal terms are more related to the local phenomena we are interested in.

Definition 14 (Decomposition of $h_{\delta}$ ). Let $F$ be a normalized periodic function in $\mathbb{R}^{n} / \mathbb{Z}^{n}$. The $\varepsilon$-periodic part $h_{\mathrm{p}, \varepsilon, \delta}[F]$ (off-diagonal part) is given by

$$
\begin{aligned}
\left\langle\hat{h}_{\mathrm{p}, \varepsilon, \delta}, \varphi\right\rangle:=- & \frac{2 b_{n, \delta}}{\varepsilon^{2 n}\|\psi\|_{2}^{2}} \sum_{\nu_{1} \neq \nu_{2}} \hat{F}\left(\nu_{1}\right) \overline{\hat{F}}\left(\nu_{2}\right) \\
& \quad \int \hat{\psi}\left(\left(\xi-\nu_{1}\right) / \varepsilon\right) \hat{\psi}\left(\left(\eta-\nu_{2}\right) / \varepsilon\right) \varphi\left(\frac{|\xi|^{2}-|\eta|^{2}}{2}\right) \frac{d \xi d \eta}{|\xi-\eta|^{n+2 \delta}},
\end{aligned}
$$

where $\varphi \in \mathcal{S}(\mathbb{R})$ is a test function. The $\varepsilon$-background part $h_{\mathrm{b}, \varepsilon, \delta}$ (diagonal part) is given by

$$
h_{\mathrm{b}, \varepsilon, \delta}[F]:=h_{\delta}\left[f_{\varepsilon}\right]-\varepsilon^{n} h_{\mathrm{p}, \varepsilon, \delta}[F] .
$$

Once we have defined the decomposition of $h_{\delta}$, we concentrate for the moment on the behavior of the $\varepsilon$-periodic part $h_{\mathrm{p}, \varepsilon, \delta}$ as $\varepsilon$ tends to zero, but first we need a definition.

Definition 15. Let $F$ be a normalized periodic function in $\mathbb{R}^{n} / \mathbb{Z}^{n}$. The periodic limit $h_{\mathrm{p}, \delta}[F]$ is given by

$$
\left\langle\hat{h}_{\mathrm{p}, \delta}, \varphi\right\rangle:=-\frac{2 b_{n, \delta}}{\|\psi\|_{2}^{2}} \sum_{\nu_{1} \neq \nu_{2}} \hat{F}\left(\nu_{1}\right) \overline{\hat{F}}\left(\nu_{2}\right) \varphi\left(\frac{\left|\nu_{1}\right|^{2}-\left|\nu_{2}\right|^{2}}{2}\right) \frac{1}{\left|\nu_{1}-\nu_{2}\right|^{n+2 \delta}},
$$

where $\varphi \in \mathcal{S}(\mathbb{R})$ is a test function. 
Lemma 16. Let $F$ be a normalized periodic function such that $\hat{F} \in \ell^{2}\left(|\nu|^{2 \delta}\right)$. If $h_{p, \varepsilon, \delta}[F]$ and $h_{p, \delta}[F]$ are the distributions in (38) and (40), respectively, then $h_{p, \varepsilon, \delta}[F]$ converges uniformly in compact sets to $h_{p, \delta}[F]$, and $\left\|\hat{h}_{p, \delta}[F]\right\|_{L^{1}} \lesssim$ 1.

Proof. The distribution $\hat{h}_{\mathrm{p}, \varepsilon, \delta}[F]$ is an integrable function. In fact, we can bound $\left|\left\langle\hat{h}_{\mathrm{p}, \varepsilon, \delta}, \varphi\right\rangle\right|$ as

$$
\left|\left\langle\hat{h}_{\mathrm{p}, \varepsilon, \delta}, \varphi\right\rangle\right| \leqslant C\|\varphi\|_{\infty},
$$

where $C$ is independent of $\varepsilon$. The same arguments used in Theorem 9 to prove (23) show that $\left\|\hat{h}_{\mathrm{p}, \varepsilon, \delta}\right\|_{L^{1}} \lesssim 1$, so there exists a measure $\mu$ and a sequence $\left\{h_{\mathrm{p}, \varepsilon_{k}, \delta}\right\}_{k}$, with $\varepsilon_{k} \rightarrow 0$, that converges weakly* to $\mu$ with $|\mu|(\mathbb{R}) \lesssim 1$.

To evaluate the integral in (38) we fix a number $R \geqslant 1$ and notice that for $(\xi, \eta)$ at distance less than $\varepsilon$ from $\left(\nu_{1}, \nu_{2}\right)$ we have two bounds: if $\left\{\max \left|\nu_{i}\right|>\right.$ $R\}$ then

$$
\begin{aligned}
& \varphi\left(\frac{|\xi|^{2}-|\eta|^{2}}{2}\right) \frac{1}{|\xi-\eta|^{n+2 \delta}}-\varphi\left(\frac{\left|\nu_{1}\right|^{2}-\left|\nu_{2}\right|^{2}}{2}\right) \frac{1}{\left|\nu_{1}-\nu_{2}\right|^{n+2 \delta}}= \\
& \mathcal{O}\left(\frac{\|\varphi\|_{\infty}}{\left|\nu_{1}-\nu_{2}\right|^{n+2 \delta}}\right)
\end{aligned}
$$

and if $\left\{\max \left|\nu_{i}\right|<R\right\}$ then

$$
\begin{aligned}
\varphi\left(\frac{|\xi|^{2}-|\eta|^{2}}{2}\right) \frac{1}{|\xi-\eta|^{n+2 \delta}}-\varphi\left(\frac{\left|\nu_{1}\right|^{2}-\left|\nu_{2}\right|^{2}}{2}\right) \frac{1}{\left|\nu_{1}-\nu_{2}\right|^{n+2 \delta}}= \\
\mathcal{O}\left(\frac{\|\varphi\|_{\infty} \varepsilon}{\left|\nu_{1}-\nu_{2}\right|^{n+2 \delta+1}}+\frac{\left\|\varphi^{\prime}\right\|_{\infty} R \varepsilon}{\left|\nu_{1}-\nu_{2}\right|^{n+2 \delta}}\right) .
\end{aligned}
$$

With these two bounds we get the following estimate for (38):

$$
\begin{aligned}
\left\langle\hat{h}_{\mathrm{p}, \varepsilon, \delta}, \varphi\right\rangle=-\frac{2 b_{n, \delta}}{\|\psi\|_{2}^{2}} \sum_{\nu_{1} \neq \nu_{2}} & \hat{F}\left(\nu_{1}\right) \overline{\hat{F}}\left(\nu_{2}\right)\left[\varphi\left(\frac{\left|\nu_{1}\right|^{2}-\left|\nu_{2}\right|^{2}}{2}\right) \frac{1}{\left|\nu_{1}-\nu_{2}\right|^{n+2 \delta}}+\right. \\
& \quad+\mathbb{1}_{\left\{\max \left|\nu_{i}\right|>R\right\}} \mathcal{O}\left(\frac{\|\varphi\|_{\infty}}{\left|\nu_{1}-\nu_{2}\right|^{n+2 \delta}}\right)+ \\
& \left.\quad+\mathbb{1}_{\left\{\max \left|\nu_{i}\right|<R\right\}} \mathcal{O}\left(\frac{\|\varphi\|_{\infty} \varepsilon}{\left|\nu_{1}-\nu_{2}\right|^{n+2 \delta+1}}+\frac{\left\|\varphi^{\prime}\right\|_{\infty} R \varepsilon}{\left|\nu_{1}-\nu_{2}\right|^{n+2 \delta}}\right)\right] \\
:=\left\langle\hat{h}_{\mathrm{p}, \delta}, \varphi\right\rangle+ & \mathrm{E}_{1}+\mathrm{E}_{2} .
\end{aligned}
$$


We bound the first error term as

$$
\begin{aligned}
\mathrm{E}_{1} & \leqslant C\|\varphi\|_{\infty} \sum_{\nu_{1}}\left|\hat{F}\left(\nu_{1}\right)\right|^{2} \sum_{\max \left|\nu_{i}\right|>R} \frac{1}{\left|\nu_{1}-\nu_{2}\right|^{n+2 \delta}} \\
& \leqslant C\|\varphi\|_{\infty} \sum_{\nu_{1}}\left|\hat{F}\left(\nu_{1}\right)\right|^{2}\left(\mathbb{1}_{\left|\nu_{1}\right|<R / 2} R^{-2 \delta}+\mathbb{1}_{\left|\nu_{1}\right|>R / 2}\right) \\
& \leqslant C\|\varphi\|_{\infty} R^{-2 \delta}\left(1+\|\hat{F}\|_{\ell^{2}(|\nu| 2 \delta)}^{2}\right)
\end{aligned}
$$

we bound the second error term as

$$
\mathrm{E}_{2} \leqslant C\left(\|\varphi\|_{\infty} \varepsilon+\left\|\varphi^{\prime}\right\|_{\infty} R \varepsilon\right)
$$

We have thus

$$
\begin{aligned}
\left\langle\hat{h}_{\mathrm{p}, \varepsilon, \delta}, \varphi\right\rangle=-\frac{2 b_{n, \delta}}{\|\psi\|_{2}^{2}} \sum_{\nu_{1} \neq \nu_{2}} \hat{F}\left(\nu_{1}\right) \overline{\hat{F}}\left(\nu_{2}\right) \varphi\left(\frac{\left|\nu_{1}\right|^{2}-\left|\nu_{2}\right|^{2}}{2}\right) \frac{1}{\left|\nu_{1}-\nu_{2}\right|^{n+2 \delta}}+ \\
+\mathcal{O}\left(\|\varphi\|_{\infty} R^{-2 \delta}+\|\varphi\|_{\infty} \varepsilon+\left\|\varphi^{\prime}\right\|_{\infty} R \varepsilon\right) .
\end{aligned}
$$

Taking $R=\varepsilon^{-\frac{1}{2}}$ we see that $\left\langle\hat{h}_{\mathrm{p}, \varepsilon, \delta}, \varphi\right\rangle \stackrel{\varepsilon \rightarrow 0}{\longrightarrow}\left\langle\hat{h}_{\mathrm{p}, \delta}, \varphi\right\rangle$ when $\varphi \in \mathcal{S}(\mathbb{R})$, which implies that $\mu=\hat{h}_{\mathrm{p}, \delta}$ is unique and that $\left\|\hat{h}_{\mathrm{p}, \delta}\right\|_{L^{1}} \lesssim 1$.

To compute $h_{\mathrm{p}, \delta}$ we set $\varphi(\tau)=e^{2 \pi i \tau t}$, and since the error terms (41) and (42) are uniform in $t$ when $|t| \leqslant T$, for any $T>0$, then we conclude that $h_{p, \varepsilon, \delta}$ converges uniformly in compact sets to $h_{\mathrm{p}, \delta}$.

The function $h_{\mathrm{p}, \delta}[F]$ is our desired extension of $h_{\delta}[f]$ to periodic functions, and we may write it as

$$
\hat{h}_{p, \delta}[F](\tau)=-\frac{2 b_{n, \delta}}{\|\psi\|_{2}^{2}} \sum_{k \in \mathbb{Z}} \delta_{\frac{k}{2}}(\tau) \sum_{\substack{\nu_{1} \neq \nu_{2} \\\left|\nu_{1}\right|^{2}-\left|\nu_{2}\right|^{2}=k}} \hat{F}\left(\nu_{1}\right) \overline{\hat{F}}\left(\nu_{2}\right) \frac{1}{\left|\nu_{1}-\nu_{2}\right|^{n+2 \delta}} .
$$

We observe that $h_{p, \delta}$ is a periodic function with period 2 .

Lemma 16 says that we can recover $h_{\mathrm{p}, \delta}[F]$ if we remove $h_{\mathrm{b}, \varepsilon, \delta}[F]$ from $h_{\delta}\left[f_{\varepsilon}\right]$, multiply by $\varepsilon^{-n}$ and then take the limit as $\varepsilon \rightarrow 0$, i.e. we can recover $h_{\mathrm{p}, \delta}[F]$ if we renormalize $h_{\delta}\left[f_{\varepsilon}\right]$.

Now we investigate the background of $h_{\delta}\left[f_{\varepsilon}\right]$, which contains the information of $\hat{h}_{\delta}\left[f_{\varepsilon}\right]$ around zero. 
Lemma 17. Let $F$ be a normalized periodic function in $\mathbb{R}^{n} / \mathbb{Z}^{n}$. If $\hat{F} \in$ $\ell^{2}\left(|\nu|^{2 \delta}\right)$, then the background $h_{b, \varepsilon, \delta}[F]$ is

$$
\begin{aligned}
h_{b, \varepsilon, \delta}(t) & =\frac{\varepsilon^{n}}{\|\psi\|_{2}^{2}} \sum_{\nu}|\hat{F}(\nu)|^{2} \int|x|^{2 \delta}\left|e^{-i \varepsilon^{2} t \hbar \Delta / 2} \psi(\varepsilon(x-t \nu))\right|^{2} d x, \\
& =\frac{\varepsilon^{-2 \delta}}{\|\psi\|_{2}^{2}} \int|x|^{2 \delta}|\psi(x)|^{2} d x+o(1),
\end{aligned}
$$

where the error term o(1) is uniform in compact sets.

If $\hat{F} \in \ell^{2}\left(|\nu|^{n+2 \delta}\right)$, then

$$
\left\|P_{>\frac{1}{4}} h_{b, \varepsilon, \delta}\right\|_{\infty}=o\left(\varepsilon^{n}\right) .
$$

If we assume further that $n=1$ and $\delta<\frac{1}{2}$, then

$$
h_{b, \varepsilon, \delta}(t)=\frac{\varepsilon^{-2 \delta}}{\|\psi\|_{2}^{2}} \int|x|^{2 \delta}|\psi(x)|^{2} d x+o(\varepsilon),
$$

where the error term $o(\varepsilon)$ is uniform in compact sets.

We can also understand (44) as a self-interaction term since the evolution of $e^{i t \hbar \Delta / 2} f_{\varepsilon}$ is

$$
e^{i \hbar \hbar \Delta / 2} f_{\varepsilon}=N_{\varepsilon}^{-1} \sum_{\nu \in \mathbb{Z}^{n}} \hat{F}(\nu) e^{2 \pi i x \cdot \nu-\pi i t|\nu|^{2}} e^{i \varepsilon^{2} t \hbar \Delta / 2} \psi(\varepsilon(x-t \nu)) .
$$

Hence, $h_{\mathrm{p}, \delta}[F]$ represents the sum of the pairwise interaction of different waves.

Equation (45) says that $\varepsilon^{2 \delta} h_{\mathrm{b}, \varepsilon, \delta}$ tends to a constant function as $\varepsilon \rightarrow 0$. Unfortunately, the rate of convergence is not fast enough, so the $\varepsilon$-periodic part $\varepsilon^{n} h_{\mathrm{p}, \varepsilon, \delta}$ may be thwarted by the noise in the limit. However, if $F$ is smooth, i.e. $\hat{F} \in \ell^{2}\left(|\nu|^{n+2 \delta}\right)$, then the high frequencies $P_{>\frac{1}{4}} h_{\mathrm{b}, \varepsilon, \delta}$ are smaller than $\varepsilon^{n} h_{\mathrm{p}, \delta}$ and, in the limit, we can think of $h_{\delta}\left[f_{\varepsilon}\right]$ as

$$
h_{\delta}\left[f_{\varepsilon}\right] \approx P_{<\frac{1}{4}} h_{\mathrm{b}, \varepsilon, \delta}+\varepsilon^{n} h_{\mathrm{p}, \delta}
$$

where $P_{<\frac{1}{4}} h_{\mathrm{b}, \varepsilon, \delta}$ is an analytic function which remains essentially constant at scale 2 , while $h_{\mathrm{p}, \delta}$ is periodic with period 2 . This representation offers the possibility of "watching" $h_{\mathrm{p}, \delta}$ numerically as tiny oscillations over a smooth background. 
Proof of Lemma 17. We begin with the proof of (44). Since $\operatorname{supp} \hat{\psi} \subset B_{1}$, we can write $h_{\delta}\left[f_{\varepsilon}\right]$ as

$$
\begin{aligned}
h_{\delta}(t)= & b_{n, \delta} \int \frac{\left|e^{-\pi i t|\xi|^{2}} \hat{f}_{\varepsilon}(\xi)-e^{-\pi i t|\eta|^{2}} \hat{f}_{\varepsilon}(\eta)\right|^{2}}{|\xi-\eta|^{n+2 \delta}} d \xi d \eta \\
= & \frac{b_{n, \delta}}{\varepsilon^{n}\|\psi\|_{2}^{2}} \sum_{\nu}|\hat{F}(\nu)|^{2} \\
& \int\left|e^{-\pi i t|\xi|^{2}} \hat{\psi}\left(\frac{\xi-\nu}{\varepsilon}\right)-e^{-\pi i t|\eta|^{2}} \hat{\psi}\left(\frac{\eta-\nu}{\varepsilon}\right)\right|^{2} \frac{d \xi d \eta}{|\xi-\eta|^{n+2 \delta}}+\varepsilon^{n} h_{p, \varepsilon, \delta}(t) \\
= & \frac{1}{\varepsilon^{n}\|\psi\|_{2}^{2}} \sum_{\nu}|\hat{F}(\nu)|^{2} \int|x|^{2 \delta}\left|e^{-i t \hbar \Delta / 2}\left(e^{2 \pi i \nu \cdot y} \psi_{\varepsilon}\right)(x)\right|^{2} d x+\varepsilon^{n} h_{p, \varepsilon, \delta}(t),
\end{aligned}
$$

where $e^{2 \pi i \nu \cdot y} \psi_{\varepsilon}(y):=\varepsilon^{n} e^{2 \pi i \nu \cdot y} \psi(\varepsilon y)$, so

$$
\left|e^{-i t \hbar \Delta / 2}\left(e^{2 \pi i \nu \cdot y} \psi_{\varepsilon}\right)(x)\right|=\varepsilon^{n}\left|\left(e^{-i \varepsilon^{2} t \hbar \Delta / 2} \psi\right)(\varepsilon(x-t \nu))\right| .
$$

We replace it above to get

$$
h_{\delta}(t)=\frac{\varepsilon^{n}}{\|\psi\|_{2}^{2}} \sum_{\nu}|\hat{F}(\nu)|^{2} \int|x|^{2 \delta}\left|\left(e^{-i \varepsilon^{2} t \hbar \Delta / 2} \psi\right)(\varepsilon(x-t \nu))\right|^{2} d x+\varepsilon^{n} h_{p, \varepsilon, \delta}(t),
$$

which implies (44) by the definition of $h_{\mathrm{b}, \varepsilon, \delta}$; see (39).

Let us define

$$
A_{\varepsilon, \nu}(t):=b_{n, \delta} \int\left|e^{-\pi i \varepsilon^{2} t|\xi|^{2}} \hat{\psi}\left(\xi-\varepsilon^{-1} \nu\right)-e^{-\pi i \varepsilon^{2} t|\eta|^{2}} \hat{\psi}\left(\eta-\varepsilon^{-1} \nu\right)\right|^{2} \frac{d \xi d \eta}{|\xi-\eta|^{n+2 \delta}}
$$

so that

$$
h_{\mathrm{b}, \varepsilon, \delta}(t)=\frac{\varepsilon^{-2 \delta}}{\|\psi\|_{2}^{2}} \sum_{\nu}|\hat{F}(\nu)|^{2} A_{\varepsilon, \nu}(t) .
$$

Since $\psi$ is real and symmetric, the Fourier transform of $A_{\varepsilon, \nu}$ is symmetric and then we can restrict ourselves to symmetric test functions, so

$$
\begin{aligned}
\left\langle\hat{A}_{\varepsilon, \nu}, \varphi\right\rangle= & b_{n, \delta} \int\left[\left|\hat{\psi}\left(\xi-\varepsilon^{-1} \nu\right)\right|^{2} \varphi(0)+\left|\hat{\psi}\left(\eta-\varepsilon^{-1} \nu\right)\right|^{2} \varphi(0)-\right. \\
& \left.-2 \varphi\left(\varepsilon^{2} \frac{|\eta|^{2}-|\xi|^{2}}{2}\right) \hat{\psi}\left(\xi-\varepsilon^{-1} \nu\right) \hat{\psi}\left(\eta-\varepsilon^{-1} \nu\right)\right] \frac{d \xi d \eta}{|\xi-\eta|^{n+2 \delta}} \\
= & \varphi(0) \int|x|^{2 \delta}|\psi|^{2} d x+ \\
& +2 b_{n, \delta} \int\left[\varphi(0)-\varphi\left(\left(\varepsilon \frac{\eta+\xi}{2}+\nu\right) \cdot \varepsilon(\eta-\xi)\right)\right] \hat{\psi}(\xi) \hat{\psi}(\eta) \frac{d \xi d \eta}{|\xi-\eta|^{n+2 \delta}}
\end{aligned}
$$


We use a test function supported in $\mathbb{R} \backslash[-a, a]$ to bound $\hat{A}_{\varepsilon, \nu}$ away from the origin as

$$
\left|\left\langle\hat{A}_{\varepsilon, \nu}, \varphi\right\rangle\right| \leqslant C \int\left|\hat{\psi}(\xi) \hat{\psi}(\eta) \varphi\left(\left(\varepsilon \frac{\eta+\xi}{2}+\nu\right) \cdot \varepsilon(\eta-\xi)\right)\right| \frac{d \xi d \eta}{|\xi-\eta|^{n+2 \delta}} .
$$

If $|\nu| \leqslant a \varepsilon^{-1} / 4$ then $\left\langle\hat{A}_{\varepsilon, \nu}, \varphi\right\rangle=0$ because $\varphi(t)=0$ when $|t| \leqslant a$. Otherwise, we change variables and bound the integral as

$$
\begin{aligned}
\left|\left\langle\hat{A}_{\varepsilon, \nu}, \varphi\right\rangle\right| & \leqslant C \int|\hat{\psi}(\xi) \hat{\psi}(\eta) \varphi((\varepsilon u+\nu) \cdot \varepsilon v)| \frac{d u d v}{|v|^{n+2 \delta}} \\
& \leqslant C\|\varphi\|_{\infty} \int_{|v|>a /(2|\nu| \varepsilon)} \frac{d v}{|v|^{n+2 \delta}} \\
& \leqslant C\|\varphi\|_{\infty}\left(\frac{|\nu| \varepsilon}{a}\right)^{2 \delta} .
\end{aligned}
$$

Hence, by Hausdorff-Young inequality $\left\|P_{>a} A_{\varepsilon, \nu}\right\|_{\infty} \lesssim_{a}(|\nu| \varepsilon)^{2 \delta}$ and then, by (48),

$$
\left\|P_{>a} h_{\mathrm{b}, \varepsilon, \delta}\right\|_{\infty} \lesssim_{a} \sum_{|\nu|>a \varepsilon^{-1} / 2}|\hat{F}(\nu)|^{2}|\nu|^{2 \delta}=o_{a}(1)
$$

to prove (45) it remains to estimate $P_{<\frac{1}{4}} h_{\mathrm{b}, \varepsilon, \delta}$. If we assume further that $\hat{F} \in \ell^{2}\left(|\nu|^{n+2 \delta}\right)$, then we can state the stronger upper bound $\left\|P_{>a} h_{\mathrm{b}, \varepsilon, \delta}\right\|_{\infty}=$ $o_{a}\left(\varepsilon^{n}\right)$, which is (46).

We turn now to the term $P_{<\frac{1}{4}} h_{\mathrm{b}, \varepsilon, \delta}$. We will prove that

$$
\left\langle\hat{h}_{\mathrm{b}, \varepsilon, \delta}, \varphi\right\rangle-\varphi(0) \varepsilon^{-2 \delta}\|\psi\|_{2}^{-2} \int|x|^{2 \delta}|\psi|^{2} d x \rightarrow 0
$$

which implies (45) after replacing $\varphi$ by the test function $\tau \mapsto \psi(\tau) \cos (2 \pi t \tau)$, where $\psi$ is a symmetric cut-off of $[-1 / 4,1 / 4]$; the bounds will be uniform in $t$ if $|t| \leqslant T$, so the convergence is uniform in compact sets.

From (49) we see that $\varepsilon^{-2 \delta}\left(\left\langle\hat{A}_{\varepsilon, \nu}, \varphi\right\rangle-\varphi(0) \int|x|^{2 \delta}|\psi|^{2} d x\right) \rightarrow 0$ as $\varepsilon \rightarrow 0$. In fact,

$$
\begin{aligned}
\varepsilon^{-2 \delta}\left|I_{\varepsilon, \nu}\right| & :=\left.\varepsilon^{-2 \delta}\left|\left\langle\hat{A}_{\varepsilon, \nu}, \varphi\right\rangle-\varphi(0) \int\right| x\right|^{2 \delta}|\psi|^{2} d x \mid \\
& \leqslant C \varepsilon^{-2 \delta} \int_{|u|,|v|<1}\left|\varphi(0)-\varphi\left(\left(\varepsilon \frac{\eta+\xi}{2}+\nu\right) \cdot \varepsilon(\eta-\xi)\right)\right| \frac{d \xi d \eta}{|\eta-\xi|^{n+2 \delta}} \\
& \leqslant C \varepsilon^{2-2 \delta}\left\|\varphi^{\prime \prime}\right\|_{\infty}
\end{aligned}
$$


and the last term tends to zero, so the claim follows.

To prove (50), and so (45), it suffices to show that $\varepsilon^{-2 \delta}|\hat{F}(\nu)|^{2}\left|I_{\varepsilon, \nu}\right|$ is uniformly dominated in $\varepsilon$ by an integrable (summable) function; recall (48) and $\|F\|_{2}=1$. To control $I_{\varepsilon, \nu}$, we change variables and bound the integral as

$$
\begin{aligned}
\left|I_{\varepsilon, \nu}\right| & \leqslant C \int_{|u|,|v|<1}|\varphi(0)-\varphi((\varepsilon u+\nu) \cdot \varepsilon v)| \frac{d u d v}{|v|^{n+2 \delta}} \\
& \lesssim\left\|\varphi^{\prime \prime}\right\|_{\infty} \varepsilon^{2}\langle\nu\rangle^{2} \int_{|v|<r} \frac{d v}{|v|^{n-2+2 \delta}}+\|\varphi\|_{\infty} \int_{r<|v|<1} \frac{d v}{|v|^{n+2 \delta}} \\
& \lesssim\left\|\varphi^{\prime \prime}\right\|_{\infty} \varepsilon^{2}\langle\nu\rangle^{2} \min \left\{r^{2(1-\delta)}, 1\right\}+\|\varphi\|_{\infty} r^{-2 \delta} \mathbb{1}_{r<1} .
\end{aligned}
$$

When $(\varepsilon\langle\nu\rangle)^{2}<\|\varphi\|_{\infty} /\left\|\varphi^{\prime \prime}\right\|_{\infty}$ we choose $r=1$ and we get

$$
\left|I_{\varepsilon, \nu}\right| \leqslant C\left\|\varphi^{\prime \prime}\right\|_{\infty} \varepsilon^{2}\langle\nu\rangle^{2} \leqslant C(\varepsilon\langle\nu\rangle)^{2 \delta}\|\varphi\|_{\infty}^{1-\delta}\left\|\varphi^{\prime \prime}\right\|_{\infty}^{\delta} .
$$

When $(\varepsilon\langle\nu\rangle)^{2}>\|\varphi\|_{\infty} /\left\|\varphi^{\prime \prime}\right\|_{\infty}$ we choose $r^{2}=\|\varphi\|_{\infty} /\left(\varepsilon^{2}\langle\nu\rangle^{2}\left\|\varphi^{\prime \prime}\right\|_{\infty}\right)$ and we get

$$
\left|I_{\varepsilon, \nu}\right| \leqslant C(\varepsilon\langle\nu\rangle)^{2 \delta}\|\varphi\|_{\infty}^{1-\delta}\left\|\varphi^{\prime \prime}\right\|_{\infty}^{\delta} .
$$

Thus, we have that $\varepsilon^{-2 \delta}|\hat{F}(\nu)|^{2}\left|I_{\varepsilon, \nu}\right| \leqslant C\|\varphi\|_{\infty}^{1-\delta}\left\|\varphi^{\prime \prime}\right\|_{\infty}^{\delta}|\hat{F}(\nu)|^{2}\langle\nu\rangle^{2 \delta}$, and (50) follows by dominated convergence.

The proof of (47) goes along the same lines, but the new hypotheses are $n=1, \delta<\frac{1}{2}$ and $\left\||\nu|^{\frac{1}{2}+\delta} \hat{F}\right\|_{\ell^{2}}<\infty$. Since $\delta<\frac{1}{2}$ we have that $\varepsilon^{-1-2 \delta}\left(\left\langle\hat{A}_{\varepsilon, \nu}, \varphi\right\rangle-\varphi(0) \int|x|^{2 \delta}|\psi|^{2} d x\right) \rightarrow 0$ as $\varepsilon \rightarrow 0$, so it suffices to show that $\varepsilon^{-1-2 \delta}|\hat{F}(\nu)|^{2}\left|I_{\varepsilon, \nu}\right|$ is uniformly dominated in $\varepsilon$ by an integrable function.

The previous bounds of $\left|I_{\varepsilon, \nu}\right|$ lead to

$$
\left|I_{\varepsilon, \nu}\right| \leqslant C\left\|\varphi^{\prime \prime}\right\|_{\infty} \varepsilon^{2}\langle\nu\rangle^{2} \leqslant C(\varepsilon\langle\nu\rangle)^{1+2 \delta}\|\varphi\|_{\infty}^{\frac{1}{2}-\delta}\left\|\varphi^{\prime \prime}\right\|_{\infty}^{\frac{1}{2}+\delta}
$$

when $(\varepsilon\langle\nu\rangle)^{2}<\|\varphi\|_{\infty} /\left\|\varphi^{\prime \prime}\right\|_{\infty}$, and

$$
\left|I_{\varepsilon, \nu}\right| \leqslant C(\varepsilon\langle\nu\rangle)^{2 \delta}\|\varphi\|_{\infty}^{1-\delta}\left\|\varphi^{\prime \prime}\right\|_{\infty}^{\delta} \leqslant C(\varepsilon\langle\nu\rangle)^{1+2 \delta}\|\varphi\|_{\infty}^{\frac{1}{2}-\delta}\left\|\varphi^{\prime \prime}\right\|_{\infty}^{\frac{1}{2}+\delta},
$$

when $(\varepsilon\langle\nu\rangle)^{2}>\|\varphi\|_{\infty} /\left\|\varphi^{\prime \prime}\right\|_{\infty}$. Therefore,

$$
\varepsilon^{-1-2 \delta}|\hat{F}(\nu)|^{2}\left|I_{\varepsilon, \nu}\right| \leqslant C\langle\nu\rangle^{1+2 \delta}\|\varphi\|_{\infty}^{\frac{1}{2}-\delta}\left\|\varphi^{\prime \prime}\right\|_{\infty}^{\frac{1}{2}+\delta} .
$$

By dominated convergence again we get (47). 
Recall that our main interest is the Talbot effect in $n=1$, so (47) in Lemma 17 provides the convenient asymptotic representation

$$
h_{\delta}(t)=\frac{\varepsilon^{-2 \delta}}{\|\psi\|_{2}^{2}} \int|x|^{2 \delta}|\psi(x)|^{2} d x+\varepsilon h_{p, \delta}(t)+o(\varepsilon),
$$

as long as $\hat{F} \in \ell^{2}\left(|\nu|^{1+2 \delta}\right)$ and $\delta<\frac{1}{2}$.

We summarize our main findings in the following theorem.

Theorem 18. Let $F$ be a normalized periodic function with period 1 in $\mathbb{R}^{n}$ recall the definition of $f_{\varepsilon}$ in (37).

If $\hat{F} \in \ell^{2}\left(|\nu|^{2 \delta}\right)$, then

$$
h_{\delta}\left[f_{\varepsilon}\right](t)=\frac{\varepsilon^{-2 \delta}}{\|\psi\|_{2}^{2}} \int|x|^{2 \delta}|\psi(x)|^{2} d x+o(1) .
$$

If $\hat{F} \in \ell^{2}\left(|\nu|^{n+2 \delta}\right)$, then

$$
h_{\delta}\left[f_{\varepsilon}\right](t)=P_{<\frac{1}{4}} h_{b, \varepsilon, \delta}(t)+\varepsilon^{n} h_{p, \delta}(t)+o\left(\varepsilon^{n}\right) .
$$

If $n=1, \delta<\frac{1}{2}$ and $\hat{F} \in \ell^{2}\left(|\nu|^{1+2 \delta}\right)$, then

$$
h_{\delta}\left[f_{\varepsilon}\right](t)=\frac{\varepsilon^{-2 \delta}}{\|\psi\|_{2}^{2}} \int|x|^{2 \delta}|\psi(x)|^{2} d x+\varepsilon h_{p, \delta}(t)+o(\varepsilon) .
$$

The error terms in all the limits are uniform in compact sets of $\mathbb{R}$.

In Figure 1 we saw how convenient is (52) to visualize $h_{\mathrm{p}, \delta}$ numerically.

\subsection{The Dirac comb}

Now that we have succeeded in defining a functional $h_{\mathrm{p}, \delta}[F]$ for a periodic function $F$, we want to pass again to the limit to study the Dirac comb, i.e. the periodic distribution $F_{D}(x):=\sum_{m \in \mathbb{Z}} \delta(x-m)$ in $\mathbb{R}$.

To approach the Dirac comb in $\mathbb{R}$ we use the function

$$
F_{\varepsilon_{1}}:=\sum_{m \in \mathbb{Z}} \varepsilon_{1}^{-1} e^{-\pi\left((x-m) / \varepsilon_{1}\right)^{2}}=\sum_{m \in \mathbb{Z}} e^{-\pi\left(\varepsilon_{1} m\right)^{2}} e^{2 \pi i x m},
$$

and define so the approximation

$$
f_{\varepsilon_{1}, \varepsilon_{2}}(x):=N_{\varepsilon_{2}}^{-1} \psi\left(\varepsilon_{2} x\right)\left\|F_{\varepsilon_{1}}\right\|_{2}^{-1} F_{\varepsilon_{1}},
$$


where $N_{\varepsilon_{2}}$ is the normalization constant of $f_{\varepsilon_{1}, \varepsilon_{2}}$. Since the periodic function $F_{\varepsilon_{1}}$, for $\varepsilon_{1}$ fixed, is smooth, then from (51) we see that $h_{\delta}\left[f_{\varepsilon_{1}, \varepsilon_{2}}\right]$ splits into a smooth background and an oscillating, periodic function $h_{\mathrm{p}, \delta}\left[F_{\varepsilon_{1}}\right]$ when $\varepsilon_{2} \rightarrow 0$.

We use (40), or (43), to see that

$$
\hat{h}_{\mathrm{p}, \delta}\left[F_{\varepsilon_{1}}\right](\tau)=-\frac{2 b_{1, \delta}}{\|\psi\|_{2}^{2}} \sum_{k \in \mathbb{Z}} \delta_{\frac{k}{2}}(\tau) \sum_{\substack{m_{1} \neq m_{2} \\\left|m_{1}\right|^{2}-\left|m_{2}\right|^{2}=k}} \hat{F}_{\varepsilon_{1}}\left(m_{1}\right) \hat{F}_{\varepsilon_{1}}\left(m_{2}\right) \frac{1}{\left|m_{1}-m_{2}\right|^{n+2 \delta}} .
$$

At this stage, we let $\varepsilon_{1}$ go to zero and take the weak limit of $\hat{h}_{\mathrm{p}, \delta}\left[F_{\varepsilon_{1}}\right]$ to get the distribution

$$
\hat{h}_{\mathrm{p}, \delta}\left[F_{D}\right](\tau):=-\frac{2 b_{1, \delta}}{\|\psi\|_{2}^{2}} \sum_{k} \delta_{\frac{k}{2}}(\tau) \sum_{\substack{m_{1} \neq m_{2} \\ m_{1}^{2}-m_{2}^{2}=k}} \frac{1}{\left|m_{1}-m_{2}\right|^{1+2 \delta}},
$$

which is our definition of periodic $h_{\delta}$ for the Dirac comb $F_{D}$. Surprisingly, $h_{\mathrm{p}, \delta}\left[F_{D}\right]$ is a pure point measure; to see this, we have to compute the coefficients of $\hat{h}_{\mathrm{p}, \delta}\left[F_{D}\right]$.

\section{Lemma 19.}

$$
\sum_{\substack{m_{1} \neq m_{2} \\ m_{1}^{2}-m_{2}^{2}=k}} \frac{1}{\left|m_{1}-m_{2}\right|^{1+2 \delta}}= \begin{cases}2 \sum_{\substack{d \mid k \\ d>0}} \frac{1}{d^{1+2 \delta}} & \text { for } k \in \mathbb{Z} \text { odd } \\ \frac{1}{2^{2 \delta} \sum_{\substack{4 \mid k \\ d>0}} \frac{1}{d^{1+2 \delta}}} & \text { for } k \equiv 0(\bmod 4) \\ 0 & \text { for } k \equiv 2(\bmod 4)\end{cases}
$$

Remark. In number theory notation, for $k$ odd the coefficients are $2 \sigma_{-1-2 \delta}(k)$, and for $k \equiv 0(\bmod 4)$ the coefficients are $2^{-2 \delta} \sigma_{-1-2 \delta}(k / 4)$.

Proof. We write $m_{1}^{2}-m_{2}^{2}=\left(m_{1}-m_{2}\right)\left(m_{1}+m_{2}\right):=d e=k$, so necessarily $d \mid k$. On the other hand, we have $m_{1}=\frac{1}{2}(e+d)$ and $m_{2}=\frac{1}{2}(e-d)$, so $d$ and $e$ have the same parity, i.e. $d \equiv e(\bmod 2)$. Consequently,

$$
\sum_{\substack{m_{1} \neq m_{2} \\ m_{1}^{2}-m_{2}^{2}=k}} \frac{1}{\left|m_{1}-m_{2}\right|^{1+2 \delta}}=2 \sum_{\substack{d \equiv e(\bmod 2) \\ d e=k, d>0}} \frac{1}{d^{1+2 \delta}}
$$

from which the Lemma follows. 


\section{Theorem $[$.}

$$
\begin{aligned}
h_{p, \delta}\left[F_{D}\right](2 t)= & -\frac{2 b_{1, \delta}}{\|\psi\|_{2}^{2}} \zeta(2(1+\delta))\left[\sum_{\substack{(p, q)=1 \\
q>0 \text { odd }}} \frac{1}{q^{2(1+\delta)}} \delta_{\frac{p}{q}}(t)-\right. \\
& \left.-\sum_{\substack{(p, q)=1 \\
q \equiv 2(\bmod 4)}} \frac{2\left(2^{1+2 \delta}-1\right)}{q^{2(1+\delta)}} \delta_{\frac{p}{q}}(t)+\sum_{\substack{(p, q)=1 \\
q \equiv 0(\bmod 4)}} \frac{2^{2(1+\delta)}}{q^{2(1+\delta)}} \delta \frac{p}{q}(t)\right], \\
:= & \sum_{\substack{(p, q)=1 \\
q>0}} \frac{a_{\delta, q}}{q^{2(1+\delta)}} \delta_{\frac{p}{q}}(t)
\end{aligned}
$$

where $\zeta(z)$ is the Riemann zeta function.

Proof. We split $\hat{h}_{\mathrm{p}, \delta}\left[F_{D}\right]$ into

$$
\begin{aligned}
\hat{h}_{\mathrm{p}, \delta}^{\mathrm{odd}}(\tau) & :=2 \sum_{k \text { odd }} \sigma_{-1-2 \delta}(k) \delta_{\frac{k}{2}}(\tau) \\
\hat{h}_{\mathrm{p}, \delta}^{\mathrm{even}}(\tau) & :=\frac{1}{2^{2 \delta}} \sum_{k \equiv 0(\bmod 4)} \sigma_{-1-2 \delta}(k / 4) \delta_{\frac{k}{2}}(\tau) .
\end{aligned}
$$

We rearrange the terms in the sum of the odd part so that

$$
\hat{h}_{\mathrm{p}, \delta}^{\text {odd }}(\tau)=2 \sum_{k \text { odd }}\left(\sum_{d \mid k} \frac{1}{d^{1+2 \delta}}\right) \delta_{\frac{k}{2}}(\tau)=2 \sum_{d>0 \text { odd }} \frac{1}{d^{1+2 \delta}} \sum_{l \text { odd }} \delta_{\frac{d l}{2}}(\tau) .
$$

The very last sum is a Dirac comb supported on the arithmetic progression $\{l$ odd $\mid d l / 2\}$, so the inverse Fourier transform of $\hat{h}_{\mathrm{p}, \delta}^{\text {odd }}$ is

$$
\begin{aligned}
h_{\mathrm{p}, \delta}^{\mathrm{odd}}(t) & =2 \sum_{\substack{d>0 \text { odd } \\
d^{2(1+\delta)}}} \frac{1}{l \in \mathbb{Z}}(-1)^{l} \delta_{\frac{l}{d}}(t) \\
& =2 \sum_{\substack{(p, q)=1 \\
q>0}} \delta_{\frac{p}{q}}(t) \sum_{\substack{d>0 \text { odd }, l \\
l / d=p / q}} \frac{(-1)^{l}}{d^{2(1+\delta)}} .
\end{aligned}
$$

Since $q \mid d$ and $p \mid l$, then

$$
h_{\mathrm{p}, \delta}^{\text {odd }}(t)=2 \sum_{\substack{(p, q)=1 \\ q>0 \text { odd }}} \delta_{\frac{p}{q}}(t) \frac{(-1)^{p}}{q^{2(1+\delta)}} \sum_{r>0 \text { odd }} \frac{1}{r^{2(1+\delta)}} .
$$


We follow a similar argument to evaluate the even part

$$
\hat{h}_{\mathrm{p}, \delta}^{\mathrm{even}}(\tau)=\frac{1}{2^{2 \delta}} \sum_{k \in \mathbb{Z}, d \mid k} \delta_{2 k}(\tau) \frac{1}{d^{1+2 \delta}}=\frac{1}{2^{2 \delta}} \sum_{d>0} \frac{1}{d^{1+2 \delta}} \sum_{l \in \mathbb{Z}} \delta_{2 d l}(\tau) ;
$$

hence, the inverse Fourier transform of $\hat{h}_{\mathrm{p}, \delta}^{\text {even }}$ is

$$
\begin{aligned}
& h_{\mathrm{p}, \delta}^{\mathrm{even}}(t)=\frac{1}{2^{1+2 \delta}} \sum_{d>0} \frac{1}{d^{2(1+\delta)}} \sum_{l \in \mathbb{Z}} \delta_{\frac{l}{2 d}}(t) \\
& =2 \sum_{\substack{(p, q)=1 \\
q>0}} \delta \frac{p}{q}(t) \sum_{\substack{d>0 \text { even }, l \\
l / d=p / q}} \frac{1}{d^{2(1+\delta)}} \\
& =2\left[\sum_{\substack{(p, q)=1 \\
q>0 \text { odd }}} \delta_{\frac{p}{q}}(t) \frac{1}{q^{2(1+\delta)}} \sum_{r>0 \text { even }} \frac{1}{r^{2(1+\delta)}}+\right. \\
& \left.+\sum_{\substack{(p, q)=1 \\
q>0 \text { even }}} \delta_{\frac{p}{q}}(t) \frac{1}{q^{2(1+\delta)}} \sum_{r>0} \frac{1}{r^{2(1+\delta)}}\right] .
\end{aligned}
$$

We sum $h_{\mathrm{p}, \delta}^{\text {even }}$ and $h_{\mathrm{p}, \delta}^{\text {odd }}$ to conclude that

$$
\begin{aligned}
h_{\mathrm{p}, \delta}(t)=-\frac{4 b_{1, \delta}}{\|\psi\|_{2}^{2}}\left[\sum_{\substack{p \text { even } \\
q>0 \text { odd }}} \frac{\zeta(2(1+\delta))}{q^{2(1+\delta)}} \delta \frac{p}{q}(t)+\right. & \sum_{\substack{(p, q)=1 \\
q>0, p \text { odds }}} \frac{-\eta(2(1+\delta))}{q^{2(1+\delta)}} \delta \frac{p}{q}(t)+ \\
& \left.+\sum_{\substack{(p, q)=1 \\
q>0 \text { even }}} \frac{\zeta(2(1+\delta))}{q^{2(1+\delta)}} \delta \frac{p}{q}(t)\right] .
\end{aligned}
$$

where $\eta(z)=-\sum_{n>0}(-1)^{n} / n^{z}=\left(1-2^{1-z}\right) \zeta(z)$ is the Dirichlet eta function. Finally, we dilate and rearrange the terms so as to get (54).

As a side remark, we notice that in higher dimensions the limit of (53) as $\varepsilon_{1} \rightarrow 0^{+}$does not exit. In the limit, the Fourier coefficients would be

$$
r_{k}=C_{\delta} \sum_{\substack{m_{1} \neq m_{2} \\\left|m_{1}\right|^{2}-\left|m_{2}\right|^{2}=k}} \frac{1}{\left|m_{1}-m_{2}\right|^{n+2 \delta}}
$$


We define the new variables $l_{1}=m_{1}-m_{2}$ and $l_{2}=m_{1}+m_{2}$ so that

$$
r_{k}=C_{\delta} \sum_{\substack{l_{1} \neq 0, l_{1} \equiv l_{2}(\bmod 2) \\ l_{1} \cdot l_{2}=k}} \frac{1}{\left|l_{1}\right|^{n+2 \delta}} .
$$

For $l_{1}$ fixed, since $l_{1} \cdot l_{2}=k$ has either no solution or infinitely many, it follows that $r_{k}=\infty$ for every integer $k$.

To study the function $h_{\mathrm{p}, \delta}\left[F_{D}\right]$ we deem it appropriate to consider its primitive

$$
H_{\delta}(t):=\int_{[0, t]} h_{\mathrm{p}, \delta}\left[F_{D}\right](2 s) d s
$$

This function is right-continuous, the limits from the left exist, has jumps at rational times and is continuous elsewhere. We think that $H_{\delta}$ can be seen as a realization of some stochastic process when $t \in[0,1)$. We do not consider $t>1$ because the derivative of a random process is almost surely non-periodic. We will review briefly some aspects of Lévy processes.

We start defining Poisson point processes, so we have to introduce point functions

$$
p: D_{p} \subset(0, \infty) \rightarrow X
$$

where $D_{p}$ is countable, and $X$ is some measure space; $X=\mathbb{R} \backslash\{0\}$ in our case. We denote by $\Pi$ the set of all point functions. To every interval $I \subset(0, \infty)$ and measurable set $U \subset X$ we assign the counting function

$$
N_{p}(I, U):=\left|\left\{t \in D_{p} \cap I \mid p(t) \in U\right\}\right| .
$$

We endow $\Pi$ with the minimal $\sigma$-field $\mathcal{B}$ generated by all the functions $p \mapsto$ $N_{p}(I, U)$.

A (stationary) Poisson point process is a random variable $\boldsymbol{p}$ from some probability space $(\Omega, \mathcal{F}, P)$ into the space of point functions $(\Pi, \mathcal{B})$, which satisfies, among other properties,

$$
\mathbf{E}\left[N_{p}(I, U)\right]:=\int_{\Omega} N_{\mathbf{p}(\omega)}(I, U) d P(\omega)=|I| n(U),
$$

where $n$ the characteristic measure of the process. We refer the reader to Ch. I.9 of [27] for details. The point function $p_{\delta}$ attached to $H_{\delta}$ represents the location and size of the jumps:

$$
p_{\delta}: \mathbb{Q} \cap[0,1) \rightarrow X=\mathbb{R} \backslash\{0\} .
$$


The following theorem shows that, in a weak sense, $\mathbf{E}\left[N_{p}(I, U)\right] \approx N_{p_{\delta}}(I, U)$ for some measure $n$ on $\mathbb{R} \backslash\{0\}$, i.e. $p_{\delta}$ resembles an outcome of some Poisson point process $\boldsymbol{p}$.

Theorem 20. For $I \subset[0,1)$, the function

$$
|N|_{p_{\delta}}(I, r):=N_{p_{\delta}}(I,(-\infty,-r] \cup[r, \infty)), \quad \text { for } r>0,
$$

satisfies the bounds

$$
\begin{array}{ll}
|N|_{p_{\delta}}(I, r) \leqslant C_{\delta}|I| r^{-1 /(1+\delta)}+1, & \text { all } r \lesssim_{\delta} 1, \\
|N|_{p_{\delta}}(I, r) \gtrsim_{\delta} \frac{|I|}{\log \left(c_{\delta} / r\right)} r^{-1 /(1+\delta)}, & \text { all } r \lesssim_{\delta}|I|^{2(1+\delta)} .
\end{array}
$$

The theorem is consequence of the following lemma.

Lemma 21. For $I \subset[0,1)$, the function

$$
M(I, N):=\mid\{p / q \in I \subset \mathbb{R} \mid q \leqslant N \text { and }(p, q)=1\} \mid, \quad \text { for } N \geqslant 1,
$$

satisfies the bounds

$$
\begin{aligned}
& M(I, N) \leqslant|I| N^{2}+1, \quad \text { all } N \geqslant 1, \\
& M(I, N) \gtrsim|I| \frac{N^{2}}{\log N}, \quad \text { all } N>2 /|I| \text {. }
\end{aligned}
$$

Proof. We arrange the rationals inside $I$ in increasing order $p_{1} / q_{1}<\cdots<$ $p_{M} / q_{M}$ and then use the fact that $p_{i+1} / q_{i+1}-p_{i} / q_{i}=1 /\left(q_{i+1} q_{i}\right)$, see Theorem 28 in [24], to get

$$
|I| \geqslant \sum_{i=1}^{M-1} \frac{1}{q_{i+1} q_{i}}>\frac{M-1}{N^{2}}
$$

which is (58).

For the lower bound, we only count fractions $p / q$ with prime denominator. Given a prime $q \leqslant N$ such that $q|I|>1$, the number of fractions $p / q \in I$ is $\geqslant q|I| / 2$, so for $N>2 /|I|$ we have

$$
M(I, N) \geqslant \frac{1}{2}|I| \sum_{|I|^{-1}<q \leqslant N} q \geqslant \frac{1}{4}|I| N \mid\{N / 2 \leqslant q \leqslant N \mid q \text { prime }\} \mid .
$$

Using the prime number theorem and the Bertrand's postulate we arrive at (59). 
We expect the bounds in the lemma can be improved, in particular, the $\log N$-loss in (59) should be removable. It is interesting to investigate the behavior of $M(I, N)$ when $N \leqslant 2 /|I|$. For example, in the interval $I=$ $(0,1 / N)$ there is no rational $p / q$ with $q \leqslant N$, so $M(I, N)$ can be zero when $q \leqslant 1 /|I|$, but $(0,1 / N)$ is a very special interval, can we do any better for other type of intervals?

Proof of Theorem 20. According to (54) the value of the point function $p_{\delta}$ at a rational time $t=p / q$ is $p_{\delta}(t)=a_{\delta, q} / q^{2(1+\delta)}$, where $\left|a_{\delta, q}\right| \sim_{\delta} 1$, so

$$
M\left(I, c_{\delta} r^{-\frac{1}{2(1+\delta)}}\right) \leqslant|N|_{p_{\delta}}(I, r) \leqslant M\left(I, C_{\delta} r^{-\frac{1}{2(1+\delta)}}\right),
$$

and the bounds in the theorem follow from Lemma 21 ,

Theorem 20 suggests that $N_{p_{\delta}}(I, U) \approx|I| n(U)$ with characteristic measure $d n(r) \approx r^{-1-1 /(1+\delta)} d r$. We can write $H_{\delta}$ in (55) in terms of $N_{p_{\delta}}$ as

$$
H_{\delta}(t)=\int_{[0, t]} \int_{\mathbb{R} \backslash\{0\}} y N_{p_{\delta}}(d s d y) .
$$

We recognize here a "realization" of an (asymmetric) $\alpha$-Lévy process with exponent $\alpha:=1 /(1+\delta)$. We ignore the compensator term because it would add a linear term in $t$, and we can always think of $H_{\delta}$ as a Lévy process with drift; see Ch. II.3-4 of [27].

This connection between $H_{\delta}$ and Lévy processes also suggests that $H_{\delta}$ behaves intermittently, with bursts at rational times with small denominator. It is worth mentioning that $\alpha$-Lévy processes, with $1<\alpha<21$ have already been studied and described as strongly intermittent; see Sec. 3.3 in [8].

Yet another evidence of intermittency lies in the variability of the Hölder exponent of $H_{\delta}$ or multifractality, which is the content of the next theorem, but first we introduce a few definitions and a lemma.

Definition 22 (Hölder exponent). Let $t_{0} \in \mathbb{R}$. A function $f$ is in $C^{l}\left(t_{0}\right)$, for $l \in \mathbb{R}_{+}$, if there is a polynomial $P_{t_{0}}$ of degree at most $\lfloor l\rfloor$ such that in a neighborhood of $t_{0}$

$$
\left|f(t)-P_{t_{0}}(t)\right| \lesssim\left|t-t_{0}\right|^{l}
$$

The Hölder exponent of $f$ at $t_{0}$ is

$$
\gamma_{f}\left(t_{0}\right):=\sup \left\{l \mid f \in C^{l}\left(t_{0}\right)\right\} .
$$

\footnotetext{
${ }^{1}$ The larger the exponent, the lower the probability of very large jumps.
} 
Definition 23 (Irrationality measure). Fix $t \in \mathbb{R}$ and let $A \subset \mathbb{R}_{+}$be the set of exponents $m \in \mathbb{R}_{+}$such that

$$
0<\left|t-\frac{p}{q}\right|<\frac{1}{q^{m}}
$$

has infinitely many solutions. The irrationality measure $\mu(t)$ of $t \in \mathbb{R}$ is

$$
\mu(t):=\sup A \text {. }
$$

If $t$ is rational, then $\mu(t)=1$; if $t$ is irrational, then by the Dirichlet's approximation theorem $\mu(t) \geqslant 2$; if $t$ is an irrational algebraic number, then $\mu(t)=2$ by Roth's theorem; and $t$ is a Liouville number if and only if $\mu(t)=\infty$.

Lemma 24. Let $t$ be an irrational number with finite $\mu(t)$ and let $\varepsilon>0$. If $P / Q$ is the fraction with the smallest denominator among all fractions $|t-p / q|<h$, for $h \ll_{\varepsilon} 1$, then $h^{-1 /(\mu+\varepsilon)}<Q$.

For $t=p_{0} / q_{0}$, if $h>0$ and $0<|t-p / q| \leqslant h$, then $q \geqslant 1 /\left(q_{0} h\right)$.

Proof. We only consider the case when $t$ is irrational. Suppose on the contrary that there is $p / q$ with $q \leqslant h^{-1 /(\mu+\varepsilon)}$ such that $0<|t-p / q|<h$, then $0<|t-p / q|<1 / q^{\mu+\varepsilon}$, but this can only happen for finitely many fractions, so taking $h \ll_{\varepsilon} 1$ we can avoid those fractions and necessarily $h^{-1 /(\mu+\varepsilon)}<Q$.

Theorem 4. Let $H_{\delta}$ be the function in (9) and set $\alpha:=1 /(1+\delta)$. Then,

$$
d_{H_{\delta}}(\gamma)= \begin{cases}\alpha \gamma, & \text { if } \gamma \in[0,1 / \alpha] \\ -\infty, & \text { if } \gamma>1 / \alpha .\end{cases}
$$

If $t$ is rational, then $\left|H_{\delta}(t+h)-H_{\delta}(t)\right| \leqslant C_{\delta}(t) h^{1+2 \delta}$ for all $h>0$.

Proof. Let $t$ be irrational. We first prove that for every $\varepsilon>0$

$$
\left|H_{\delta}(t+h)-H_{\delta}(t)\right| \leqslant C|h|^{2(1+\delta) /(\mu+\varepsilon)}, \quad \text { for } h \ll_{t, \varepsilon} 1,
$$

so $\gamma_{H_{\delta}}(t) \geqslant 2(1+\delta) / \mu$ if $2 \leqslant \mu(t) \leqslant \infty$; recall Def. 22 .

We assume that $h>0$, the other case being similar. We integrate by parts to write the difference as

$$
\begin{aligned}
H_{\delta}(t+h)-H_{\delta}(t) & =\int_{\mathbb{R} \backslash\{0\}} y N_{p_{\delta}}(I, d y) \\
& =\int_{0}^{\infty}\left[N_{p_{\delta}}(I,[y, \infty))-N_{p_{\delta}}(I,[-y,-\infty))\right] d y .
\end{aligned}
$$


Among all $p / q \in I=(t, t+h]$, let $P / Q$ be the rational with the smallest denominator, so

$$
\begin{aligned}
& H_{\delta}(t+h)-H_{\delta}(t)=\frac{a_{\delta, Q}}{Q^{2(1+\delta)}}+ \\
& \qquad \int_{0}^{\left|a_{\delta, Q}\right| / Q^{2(1+\delta)}}\left[N_{p_{\delta}}(I,[y, \infty))-N_{p_{\delta}}(I,[-y,-\infty))-\frac{a_{\delta, Q}}{\left|a_{\delta, Q}\right|}\right] d y \\
& \quad=\frac{a_{\delta, Q}}{Q^{2(1+\delta)}}+J_{1} .
\end{aligned}
$$

To control the integral $J_{1}$ we recall the definition of $|N|_{p_{\delta}}(I, r)$ in (56) and write

$$
\left|J_{1}\right| \leqslant \int_{0}^{\left|a_{\delta, Q}\right| / Q^{2(1+\delta)}}|N|_{p_{\delta}}(I, r)-1 d r=\int_{0}^{\left|a_{\delta, Q *}\right| / Q_{*}^{2(1+\delta)}}|N|_{p_{\delta}}(I, r)-1 d r,
$$

where $Q_{*}>Q$ is the next to the smallest denominator in $I:=(t, t+h]$. Since $P / Q$ and $P_{*} / Q_{*}$ have to be successive in a Farey sequence, then $1 / Q_{*}^{2}<$ $1 /\left(Q Q_{*}\right)<h$, so, using (57), we have that $\left|J_{1}\right| \leqslant C_{\delta} h Q_{*}^{-2 \delta} \leqslant C_{\delta} h^{1+\delta}$. Hence,

$$
H_{\delta}(t+h)-H_{\delta}(t)=a_{\delta, Q} / Q^{2(1+\delta)}+\mathcal{O}\left(h^{1+\delta}\right)
$$

and from Lemma 24 we get (61), so $\gamma_{H_{\delta}}(t) \geqslant 2(1+\delta) / \mu$.

To see that the exponent in (61) is the best possible when $2<\mu(t) \leqslant \infty$, let $\left\{q_{i}\right\}_{i}$ be an infinite list of numbers such that $\left|t-p_{i} / q_{i}\right|<1 / q_{i}^{\mu-\varepsilon}$ for some $\varepsilon>0$. If we take $h_{i}=1 / q_{i}^{\mu-\varepsilon}$, then the smallest denominator in $\left(t, t+h_{i}\right]$ is $Q \leqslant q_{i}$. By (63),$\left|H_{\delta}(t+h)-H_{\delta}(t)\right| \gtrsim h_{i}^{2(1+\delta) /(\mu-\varepsilon)}$ if $h_{i} \ll 1$.

Now we show by contradiction that for every $\gamma>1+\delta$ the set of numbers $t$ for which $H_{\delta} \in C^{\gamma}(t)$ is empty. Suppose there is a polynomial $P_{t}(h)=$ $H_{\delta}(t)+h R_{t}(h)$ of degree less than $\lfloor\gamma\rfloor$ such that

$$
\left|H_{\delta}(t+h)-H_{\delta}(t)-h R_{t}(h)\right| \leqslant C h^{\gamma}, \quad \text { for all }|h| \ll 1 .
$$

By the Dirichlet's approximation theorem we can choose a sequence $\left\{q_{i}\right\}$ such that $\left|t-p_{i} / q_{i}\right|<1 / q_{i}^{2}$. Set $h_{i}=p_{i} / q_{i}-t$ so that $h_{i}<1 / q_{i}^{2}$. Let us write $H_{\delta}(s \pm):=\lim _{\varepsilon \rightarrow 0^{+}} H_{\delta}(s \pm \varepsilon)$, and notice that

$$
H_{\delta}\left(t+h_{i}+\right)-H_{\delta}(t)=H_{\delta}\left(t+h_{i}-\right)-H_{\delta}(t)+\frac{a_{\delta, q_{i}}}{q_{i}^{2(1+\delta)}} .
$$


From (64) and (65) we see that

$$
\left|H_{\delta}\left(t+h_{i}+\right)-H_{\delta}(t)-h_{i} R_{t}\left(h_{i}\right)\right| \leqslant C h_{i}^{\gamma},
$$

and that

$$
\left|H_{\delta}\left(t+h_{i}-\right)-H_{\delta}(t)+\frac{a_{\delta}}{q_{i}^{2(1+\delta)}}-h_{i} R_{t}\left(h_{i}\right)\right| \geqslant \frac{\left|a_{\delta, q_{i}}\right|}{q_{i}^{2(1+\delta)}}-C h_{i}^{\gamma} .
$$

Since $h_{i}^{1+\delta}<1 / q_{i}^{2(1+\delta)}$ and $\left|a_{\delta, q}\right| \simeq_{\delta} 1$, then we conclude that

$$
h_{i}^{1+\delta}<\frac{1}{q_{i}^{2(1+\delta)}} \leqslant C_{\delta} h_{i}^{\gamma}, \quad \text { where } h_{i} \rightarrow 0,
$$

which is a contradiction when $\gamma>1+\delta$, so $d_{H_{\delta}}(\gamma)=-\infty$ in this case.

Up to now, we know that for $2 \leqslant \mu(t) \leqslant \infty$ there is a sequence $h_{i} \rightarrow 0$ such that $\left|h_{i}\right|^{2(1+\delta) /(\mu-\varepsilon)} \lesssim\left|H_{\delta}\left(t+h_{i}\right)-H_{\delta}(t)\right| \lesssim\left|h_{i}\right|^{2(1+\delta) /(\mu+\varepsilon)}$, so necessarily $\gamma_{H_{\delta}}(t)=2(1+\delta) / \mu$ as long as $2(1+\delta) / \mu \neq 1$. To prove the theorem we still need to settle the case $2(1+\delta) / \mu=1$.

From (63) we see that $H_{\delta}(t+h)-H_{\delta}(t)-A h=a_{\delta, Q} / Q^{2(1+\delta)}-A h+\mathcal{O}\left(h^{1+\delta}\right)$, where $A$ is any constant. Again, $\left|H_{\delta}(t+h)-H_{\delta}(t)-A h\right| \lesssim|h|^{2(1+\delta) /(\mu+\varepsilon)}$, but now, to see that this is best possible, we use Dirichlet's theorem to find a sequence $\left\{q_{i}\right\}$ such that $\left|t-p_{i} / q_{i}\right|<1 / q_{i}^{2}$. We choose $h_{i}=1 / q_{i}^{2}$ and notice that $Q=q_{i}$ is the smallest denominator among all fractions in $\left(t, t+h_{i}\right]$. Indeed, if $Q<q_{i}$, then we can consider the Farey sequence up to the denominator $q_{i}$. Now let $p / q \in\left(t, t+h_{i}\right]$ be contiguous to $p_{i} / q_{i}$ and satisfy $q<q_{i}$ so that $\left|p_{i} / q_{i}-p / q\right|=1 /\left(q q_{i}\right)<h_{i}=1 / q_{i}^{2}$, which implies $q>q_{i}$, a contradiction. Thus, the linear term dominates and $\left|H_{\delta}\left(t+h_{i}\right)-H_{\delta}(t)-A h_{i}\right| \gtrsim\left|h_{i}\right|$, which implies $\gamma_{H_{\delta}}(t)=1$.

When $t$ is rational $\gamma_{H_{\delta}}(t)=0$, but we can still measure the Hölder exponent from the right using (62), (157) and Lemma 24.

To conclude the theorem we use a result of Güting [22], which asserts that the Hausdorff dimension of the set of numbers with irrationality $\mu$ is

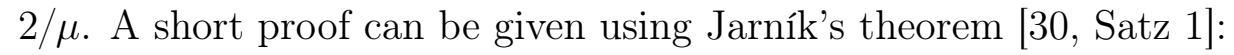

$$
\begin{gathered}
\mathcal{H}^{2 / \mu}\left(W_{\mu}\right)=\infty, \\
W_{\mu}=\left\{t|| t-\frac{p}{q} \mid<\frac{1}{q^{\mu}} \text { for infinitely many fractions } p / q\right\} ;
\end{gathered}
$$

see also [5]. The set of numbers where $H_{\delta}$ has Hölder exponent $\gamma \leqslant 1+\delta:=$ $1 / \alpha$ coincides with the set of numbers with irrationality $2(1+\delta) / \gamma$, and the dimension of the latter is $\gamma /(1+\delta)$, which is (60). 


\section{References}

[1] Mikel Agirre. The Schrödinger equation and Uncertainty Principles. PhD thesis, University of the Basque Country UPV/EHU, 2020.

[2] Valeria Banica and Luis Vega. Riemann's non-differentiable function and the binormal curvature flow. Preprint arXiv:2007.07184 [math.AP], 2020 .

[3] William Beckner. Pitt's inequality and the uncertainty principle. Proc. Am. Math. Soc., 123(6):1897-1905, 1995.

[4] John J. Benedetto and Hans P. Heinig. Weighted Fourier inequalities: New proofs and generalizations. J. Fourier Anal. Appl., 9(1):1-37, 2003.

[5] Victor Beresnevich, Detta Dickinson, and Sanju Velani. Sets of exact 'logarithmic' order in the theory of Diophantine approximation. Math. Ann., 321(2):253-273, 2001.

[6] M. V. Berry. Quantum fractals in boxes. J. Phys. A, 29(20):6617-6629, 1996.

[7] M. V. Berry and S. Klein. Integer, fractional and fractal Talbot effects. J. Modern Opt., 43(10):2139-2164, 1996.

[8] Joan Bruna, Stéphane Mallat, Emmanuel Bacry, and Jean-François Muzy. Intermittent process analysis with scattering moments. Ann. Stat., 43(1):323-351, 2015.

[9] Gong Chen and Peter J. Olver. Numerical simulation of nonlinear dispersive quantization. Discrete Contin. Dyn. Syst., 34(3):991-1008, 2014.

[10] Zhen-Qing Chen, Panki Kim, and Renming Song. Heat kernel estimates for the Dirichlet fractional Laplacian. J. Eur. Math. Soc. (JEMS), 12(5):1307-1329, 2010.

[11] Vasilis Chousionis, M. Burak Erdoğan, and Nikolaos Tzirakis. Fractal solutions of linear and nonlinear dispersive partial differential equations. Proc. Lond. Math. Soc. (3), 110(3):543-564, 2015. 
[12] Michael G. Cowling and John F. Price. Bandwidth versus time concentration: the Heisenberg-Pauli-Weyl inequality. SIAM J. Math. Anal., 15(1):151-165, 1984.

[13] Francisco de la Hoz and Luis Vega. Vortex filament equation for a regular polygon. Nonlinearity, 27(12):3031-3057, 2014.

[14] M. B. Erdoğan and G. Shakan. Fractal solutions of dispersive partial differential equations on the torus. Selecta Math. (N.S.), 25(1):Paper No. 11, 26, 2019.

[15] M. B. Erdoğan and N. Tzirakis. Talbot effect for the cubic non-linear Schrödinger equation on the torus. Math. Res. Lett., 20(6):1081-1090, 2013.

[16] M. Burak Erdoğan and Nikolaos Tzirakis. Dispersive partial differential equations. Wellposedness and applications, volume 86. Cambridge: Cambridge University Press, 2016.

[17] L. Escauriaza, C. E. Kenig, G. Ponce, and L. Vega. Uniqueness properties of solutions to Schrödinger equations. Bull. Am. Math. Soc., New Ser., 49(3):415-442, 2012.

[18] Gerald B. Folland and Alladi Sitaram. The uncertainty principle: A mathematical survey. J. Fourier Anal. Appl., 3(3):207-238, 1997.

[19] Rupert L. Frank. Eigenvalue bounds for the fractional Laplacian: a review. In Recent developments in nonlocal theory, pages 210-235. Berlin: De Gruyter Open, 2018.

[20] Rupert L. Frank and Enno Lenzmann. Uniqueness of non-linear ground states for fractional Laplacians in $\mathbb{R}$. Acta Math., 210(2):261-318, 2013.

[21] Uriel Frisch. Turbulence. Cambridge University Press, Cambridge, 1995. The legacy of A. N. Kolmogorov.

[22] R. Güting. On Mahler's function $\theta_{1}$. Mich. Math. J., 10:161-179, 1963.

[23] J. H. Hannay and M. V. Berry. Quantization of linear maps on a torusFresnel diffraction by a periodic grating. Phys. D, 1(3):267-290, 1980. 
[24] G. H. Hardy and E. M. Wright. An introduction to the theory of numbers. Edited and revised by D. R. Heath-Brown and J. H. Silverman. With a foreword by Andrew Wiles. 6th ed. Oxford: Oxford University Press, 6th ed. edition, 2008.

[25] Hidenori Hasimoto. A soliton on a vortex filament. J. Fluid Mech., 51(3):477-485, 1972.

[26] I. I. Hirschman. A note on entropy. Am. J. Math., 79:152-156, 1957.

[27] Nobuyuki Ikeda and Shinzo Watanabe. Stochastic differential equations and diffusion processes. 2nd ed, volume 24. Amsterdam etc.: NorthHolland; Tokyo: Kodansha Ltd., 2nd ed. edition, 1989.

[28] Stéphane Jaffard. The multifractal nature of Lévy processes. Probab. Theory Relat. Fields, 114(2):207-227, 1999.

[29] Philippe Jaming. Nazarov's uncertainty principles in higher dimension. J. Approx. Theory, 149(1):30-41, 2007.

[30] Vojtěch Jarník. Über die simultanen diophantischen Approximationen. Math. Z., 33(1):505-543, 1931.

[31] Kamil Kaleta and Tadeusz Kulczycki. Intrinsic ultracontractivity for Schrödinger operators based on fractional Laplacians. Potential Anal., 33(4):313-339, 2010.

[32] J. Nahas and G. Ponce. On the persistent properties of solutions to semi-linear Schrödinger equation. Commun. Partial Differ. Equations, 34(10):1208-1227, 2009.

[33] Michael Reed and Barry Simon. Methods of modern mathematical physics. IV: Analysis of operators. New York - San Francisco - London: Academic Press. XV, 396 p. \$ 34.00; £22.10 (1978)., 1978.

[34] Igor Rodnianski. Fractal solutions of the Schrödinger equation. In Nonlinear PDE's, dynamics and continuum physics (South Hadley, MA, 1998), volume 255 of Contemp. Math., pages 181-187. Amer. Math. Soc., Providence, RI, 2000. 
[35] E. Stein. Singular integrals and differentiability properties of functions. Princeton Mathematical Series, No. 30. Princeton University Press, Princeton, N.J., 1970.

[36] E. Stein. Harmonic analysis: real-variable methods, orthogonality, and oscillatory integrals, volume 43 of Princeton Mathematical Series. Princeton University Press, Princeton, NJ, 1993. With the assistance of Timothy S. Murphy, Monographs in Harmonic Analysis, III.

[37] Stefan Steinerberger. Fourier uncertainty principles, scale space theory and the smoothest average. Preprint arXiv:2005.01665 [math.CA], 2020.

[38] Henry F. Talbot. Facts relating to optical science. No. IV. Philos. Mag., 9(56):401-407, 1836. 
$|\xi|>2$

$\zeta\left(\left(|\eta|^{2}-|\xi|^{2}\right) / 2\right) \neq 0$

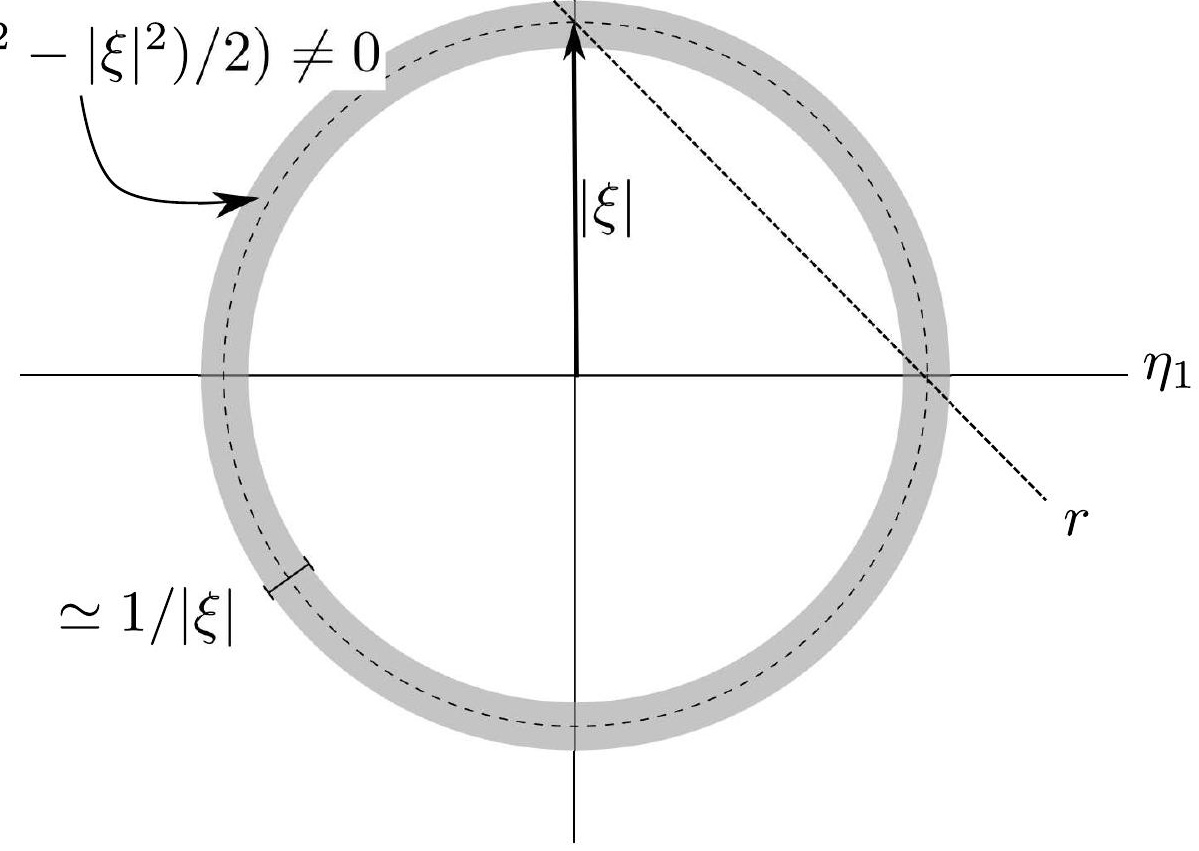

